\title{
Itinéraires thérapeutiques des adolescentes pendant la grossesse, l'accouchement et la période post-partum dans la zone d'intervention du projet
}

\author{
Gisele Kaboré \\ Population Council \\ Aicha Tamboura/Diawara \\ Assita Nacanabo Diallo \\ Abdoulaye Karama
}

Follow this and additional works at: https://knowledgecommons.popcouncil.org/departments_sbsr-pgy

Part of the Demography, Population, and Ecology Commons, Family, Life Course, and Society Commons, Gender and Sexuality Commons, International Public Health Commons, Maternal and Child Health Commons, and the Medicine and Health Commons How does access to this work benefit you? Let us know!

\section{Recommended Citation}

Kaboré, Gisele, Aicha Tamboura/Diawara, Assita Nacanabo Diallo, and Abdoulaye Karama. 2009.

"Itinéraires thérapeutiques des adolescentes pendant la grossesse, l'accouchement et la période postpartum dans la zone d'intervention du projet," Rapport final. Ouagadouogu: Population Council. 


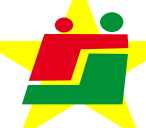

Projet : " Eliminer le mariage des enfants au Burkina : un plan pour la protection, le renforcement et l'action communautaire ".
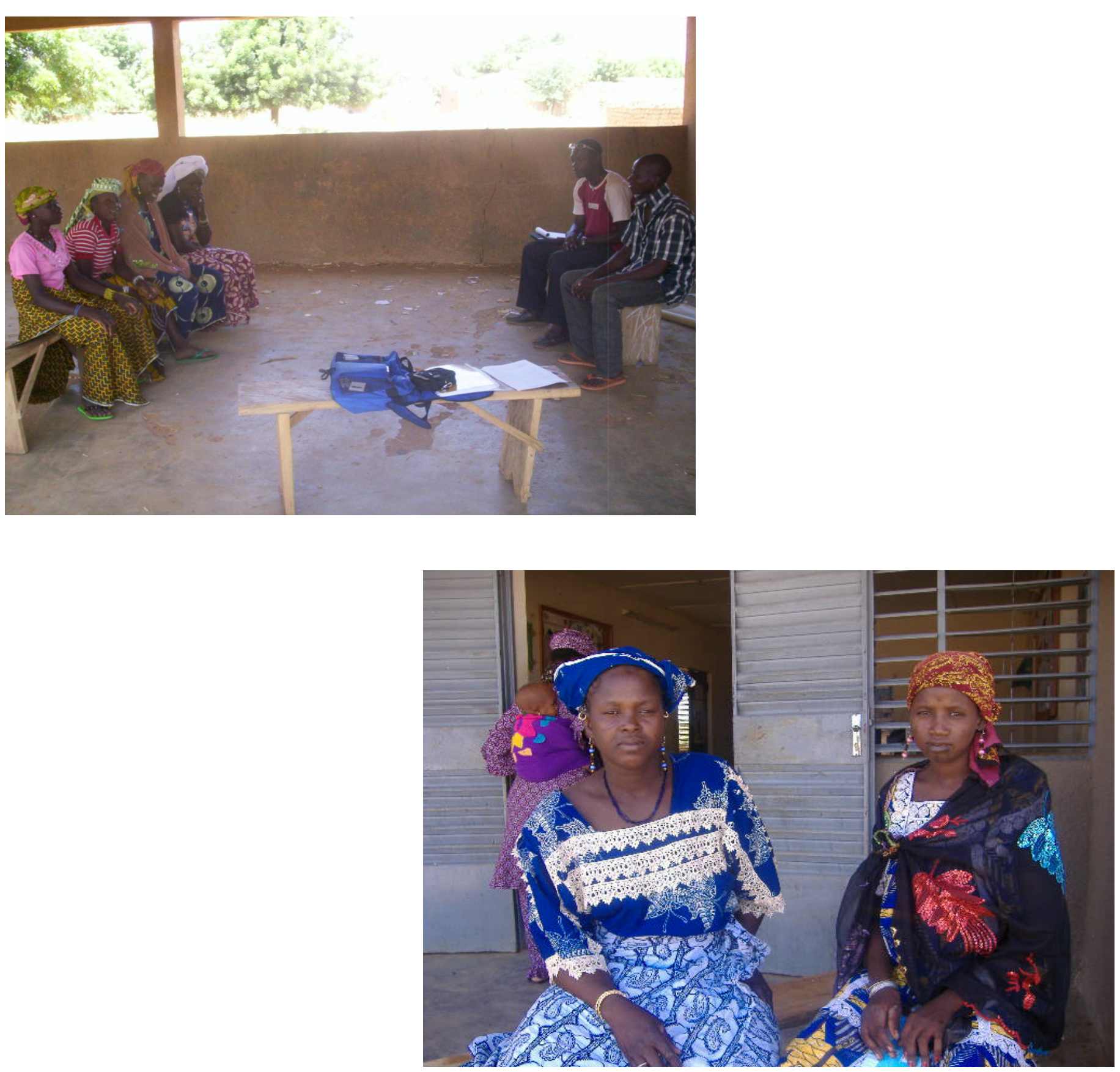

ITINERAIRES THERAPEUTIQUES DES ADOLESCENTES PENDANT LA GROSSESSE, L'ACCOUCHEMENT ET LA PERIODE POST-PARTUM DANS LA ZONE D'INTERVENTION DU PROJET

A Population Counci 


\section{PHOTOS DE COUVERTURE}

PHOTO 2: Focus Group avec des adolescentes mariées, à Yakouta.

PHOTO 1: Adolescentes venues pour la CPN

\section{EQUIPE DE RECHERCHE}

Population Council

Gisèle Kaboré

\section{Consultants}

Aïcha Tamboura/Diawara

Assita Nacanabo Diallo

Abdoulaye Karama 


\section{SOMMAIRE}

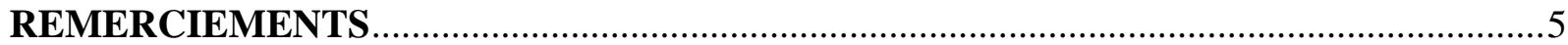

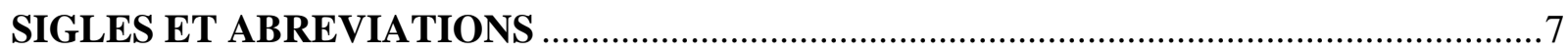

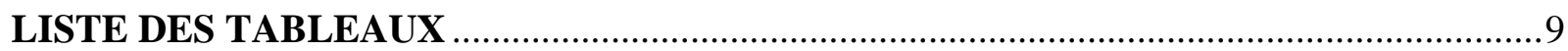

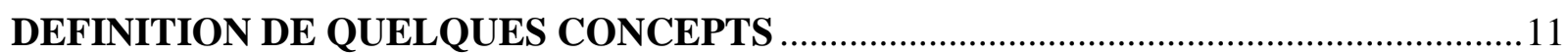

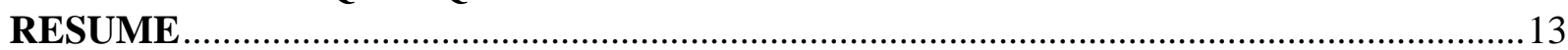

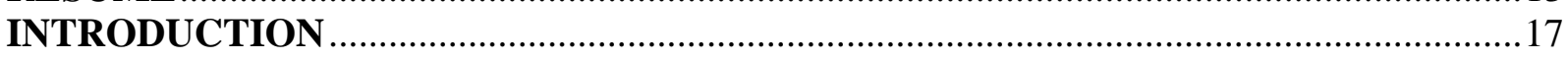

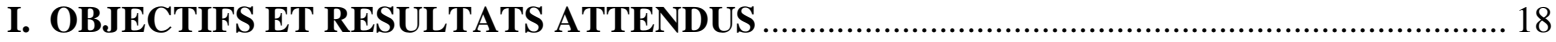

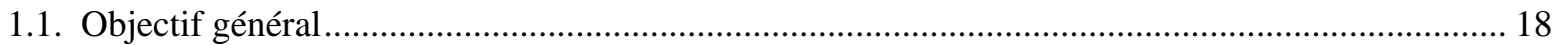

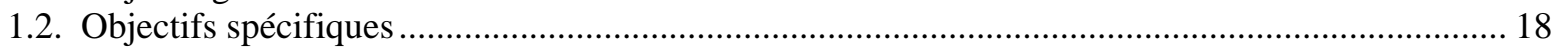

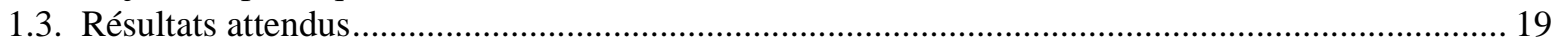

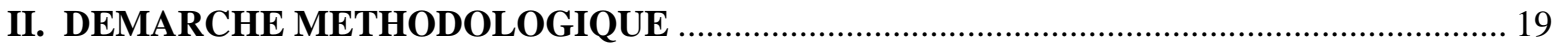

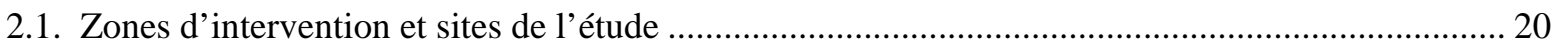

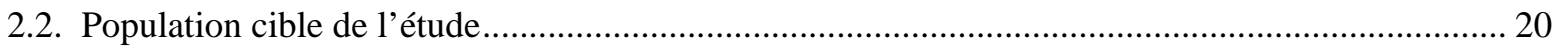

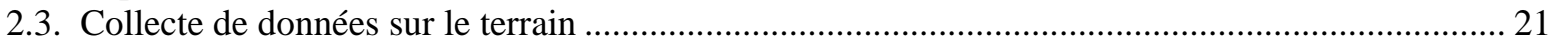

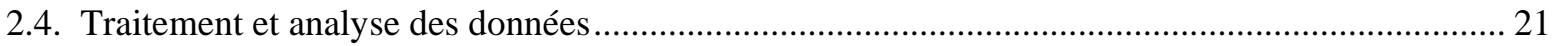

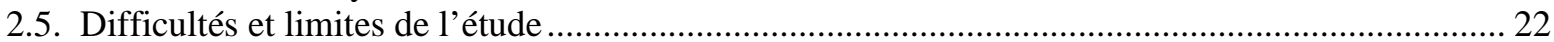

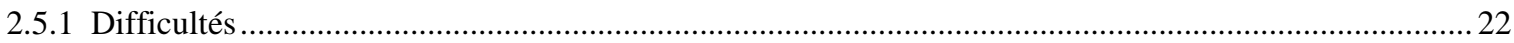

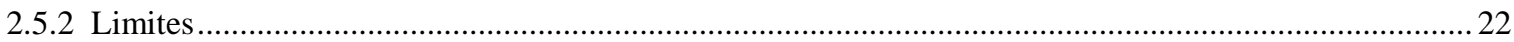

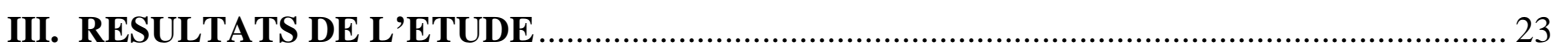

3.1. Caractéristiques sociodémographiques des enquêtées ................................................................... 23

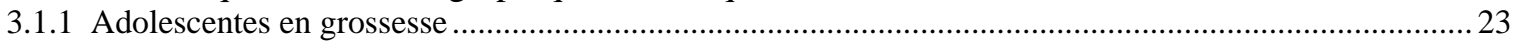

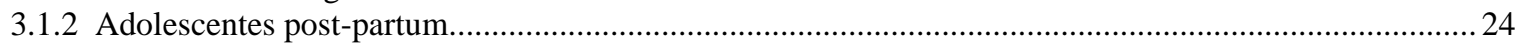

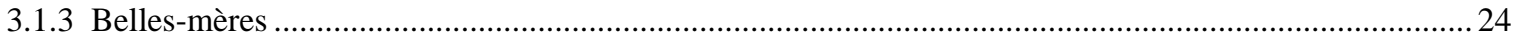

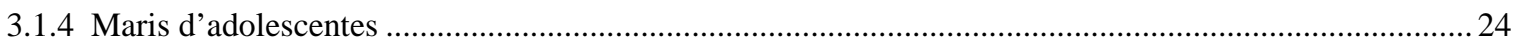

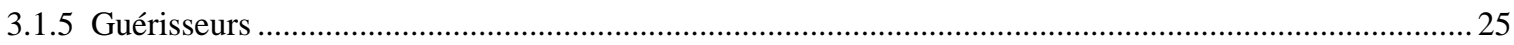

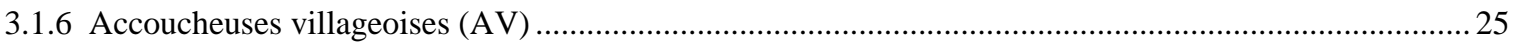

3.2. Sexualité et représentation sociale de la maternité dans les zones de l'étude............................... 25

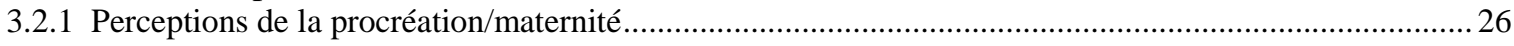

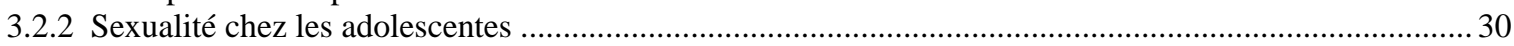

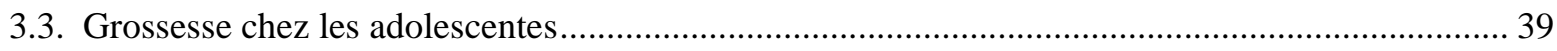

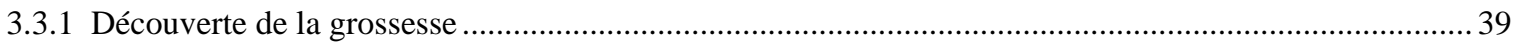

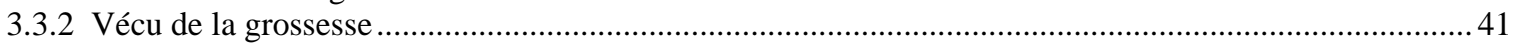

3.4. Itinéraires thérapeutiques des adolescentes pendant la grossesse .................................................. 45

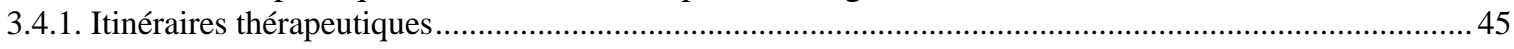

3.4.2 Connaissances sur la grossesse et besoins en informations .................................................................... 46

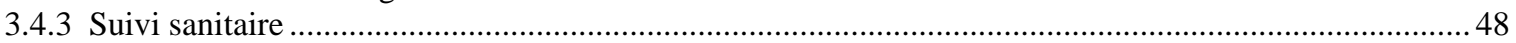

3.4.4 Gestion de la grossesse des adolescentes et prise de décisions............................................................ 49

3.4.5 Appréciation des prestations des services de santé par les adolescentes ...................................................52

3.4.6 Besoins des adolescentes en matière de soins pendant la grossesse ……………………............... 53

3.5. Itinéraires thérapeutiques des adolescentes pour l'accouchement ................................................... 54

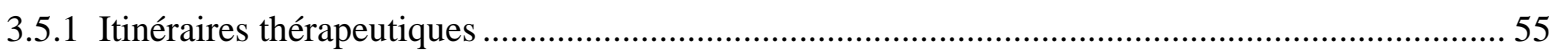




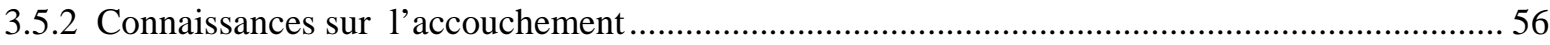

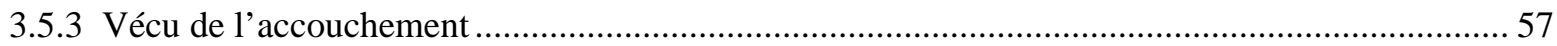

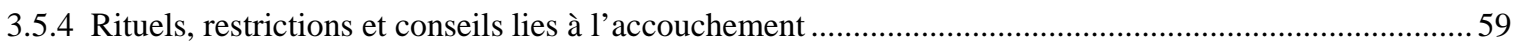

3.5.5 Profils et rôles des personnes ressources dans la gestion de l'accouchement............................................59

3.6. Itinéraires thérapeutiques des adolescentes pendant la période post-partum .................................. 62

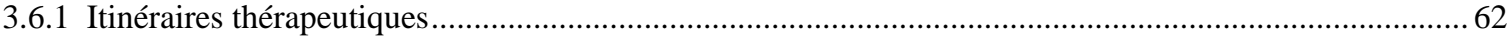

3.6.2 Prise en charge de l'adolescente durant la période post-partum ..................................................... 63

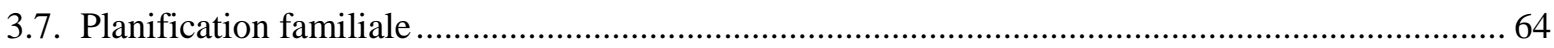

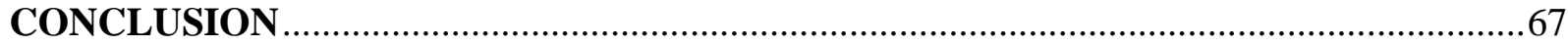

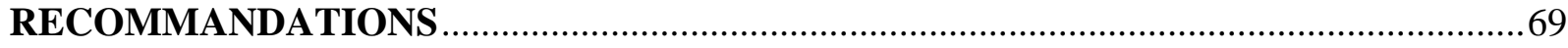

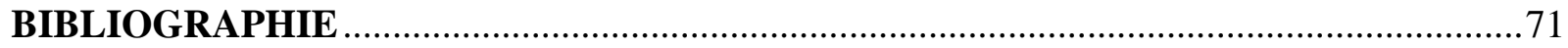


Le Population Council et l'équipe de recherche tient à adresser ses remerciements à tous ceux qui de par leur engagement, ont contribué à la réalisation de cette étude.

Nous exprimons notre gratitude aux autorités administratives (Gouverneurs, HautsCommissaires, Préfets, et Maires) des cinq régions d'intervention du Projet pour toutes les actions de facilitation et de mobilisation des populations cibles de cette étude.

Nos remerciements vont également à l'endroit des chefs d'établissements secondaires et des populations cibles enquêtées dans les cinq régions pour leur disponibilité et leur sensibilité au sujet, ce qui a facilité la collecte des données et contribué à la qualité des réponses.

Nous remercions aussi les guides et les interprètes pour leurs dévouements qui ont contribué de façon significative à la réussite de l'enquête, souvent dans des conditions difficiles. Leur détermination et leur savoir-faire ont été décisifs dans la qualité des informations recueillies.

L'exécution de l'enquête a été facilitée par la disponibilité et la collaboration des responsables de structures et d'institutions. A ce titre, nos remerciements vont à l'endroit :

- des responsables régionaux, provinciaux et départementaux (DRASSN, DRS, DRESSRS) des Régions du Sahel, du Centre-Nord, de l'Est, du Centre-Est, du Centre-Sud qui ont accueilli l'équipe de chercheurs sur le terrain et leur ont assuré l'accès aux sources d'informations ;

- des coordonnateurs et responsables d'ONG et d'Associations pour la facilitation des activités au niveau des zones d'étude ;

- des conseillers de villages qui ont permis de rencontrer les populations et ont facilité le travail de collecte des données ;

- des populations cibles des sites de collecte des informations dans les cinq régions : Dori, Saouga, Tibilindi, Noaka, Kaya, Tenkodogo, Bissiga, Ouargaye, Bogandé, Manni, Manga et Gogo. Leur accueil et leur disponibilité à répondre aux questions ont facilité le recueil des informations.

Cette liste n'est certainement pas exhaustive car plusieurs autres personnes ont également apporté une précieuse contribution pour la réalisation de ce travail. Qu'elles trouvent ici l'expression de notre sincère reconnaissance. 

ADEP

ANASIT

AV

CAWTAR

CFED

CPF

CPN

CSPS

DRASSN

DRESSRS

DRS

EDS

GONO

ICP

IST/SIDA

OMS

ONG

PF

PSP

SONU

SR

SVT

UNICEF

UNFPA
Association D’appui et d'Eveil Pugsada

Analyse Situationnelle

Accoucheuse villageoise

Centre de la Femme Arabe pour la Formation et la Recherche

Centre Français sur la Population et le Développement

Code des Personnes et de la Famille

Consultation Prénatale

Centre de Santé et de Promotion Sociale

Direction Régionale de l’Action Sociale et de la Solidarité Nationale

Direction Régionale des Enseignements Secondaires, Supérieurs et de la Recherche Scientifique

Direction Régionale de la Santé

Enquête Démographique et de Santé

Gonococcie

Infirmier Chef de Poste

Infection Sexuellement Transmissible/Syndrome d'Immunodéficience Acquise

Organisation Mondiale de la Santé

Organisation Non Gouvernementale

Planification Familiale

Poste de Santé Primaire

Soin Obstétrical Néonatal d’Urgence

Santé de la Reproduction

Science de la Vie et de la Terre

Fonds des Nations Unies pour l'Enfance

Fonds des Nations Unies pour la Population 

Tableau $\mathbf{N}^{\circ} 1$ Répartition des sites de l'étude par région

Tableau $\mathbf{N}^{\circ} 2$ Liste des outils de collecte par type d'information recherché

Tableau $\mathbf{N}^{\circ} 3$ Nombre d'entretiens individuels réalisés par région

Tableau $\mathbf{N}^{\circ} 4 \quad$ Nombre de focus group réalisés par région. 

Itinéraire thérapeutique : C'est un processus dynamique non linéaire de recherche de soin. Autrement dit, c'est une démarche qui consiste à obtenir des soins dans la cellule familiale, dans la communauté et auprès des structures modernes de santé. Dans le cadre de cette étude, ce concept a été utilisé pour désigner le parcours emprunté par les adolescentes pendant leur grossesse, leur accouchement et la période post-partum dans le cercle familiale, la communauté et dans les structures socio- sanitaires.

Grossesse précoce : Ce terme est utilisé pour designer la grossesse de l’adolescente. La précocité est déterminée par l'âge de 10-19 ans défini dans les termes de référence de l'étude.

Post-partum : C'est la période après l'accouchement. Elle va du jour de l'accouchement jusqu'au $40^{\text {ème }}$ jour. 

Le Population Council a réalisé d’octobre 2008 à février 2009, une étude sur « les itinéraires thérapeutiques des adolescentes pendant la grossesse, l'accouchement et la période post-partum » dans cinq régions du Burkina : Sahel, Centre-Nord, Centre-Est, Est, Centre-Sud.

Il s'agit d'une étude qualitative, exploratoire qui a permis d’identifier et de décrire les itinéraires thérapeutiques des adolescentes en grossesse et post-partum en vue de déceler les obstacles liés à la fréquentation des centres de santé dans le suivi de la grossesse, de l'accouchement assisté et des soins aux nouveau-nés.

Douze sites (urbains et ruraux) ont été retenus pour la collecte des données, où cohabitent les groupes ethniques Mossi, Peuhl, Bissa et Gourmantché. Les groupes cibles concernés par l'étude sont : les adolescentes mariées ou non en grossesse et post-partum et les adolescentes célibataires non en grossesse et non post-partum de 10 à 19 ans, les conjoints des adolescentes mariées, les belles-mères des adolescentes mariées en grossesse et post-partum, les personnes ressources de la communauté intervenant dans la gestion de la grossesse des adolescentes.

Deux techniques de collecte des données ont été utilisées: les entretiens individuels et les focus group.

D’une façon générale, la démarche méthodologique adoptée a concerné la revue documentaire et la collecte de données sur le terrain à l'aide des guides d'entretien individuel et des guides de discussion de groupes.

Au total, 222 personnes dont 122 femmes ont été interrogées individuellement et 209 adolescentes en focus group.

Les résultats de la revue documentaire ont fait ressortir une sexualité très active des adolescents et adolescentes, ce qui a été confirmé par les résultats de la présente recherche par rapport à la prédominance d'une sexualité précoce chez les adolescentes mariées ou non dans les cinq régions visitées. Les données de l'enquête montrent que la moyenne d’âge au premier rapport sexuel pour les adolescentes enquêtées se situe à 15,9 ans.

Un aperçu de la sexualité des adolescentes dans les différentes régions de l'étude montre les mêmes caractéristiques qui sont ponctuées par une extrême précocité, une certaine ampleur malgré son caractère tabou, une recrudescence des grossesses précoces et/ou non désirées. Cette sexualité est causée aussi par les pratiques néfastes comme l'enlèvement des filles, le mariage précoce et forcé. La majorité des adolescentes mariées ont eu leur premier rapport sexuel dans le mariage tandis que les non mariés l'ont vécu lors des occasions et des lieux divers de festivités. Quelle que soit la manière dont cette sexualité est vécue, elle est sujette à des risques du fait de la sous-information de la majorité des adolescentes sur la SR.

Les entretiens individuels ont fait ressortir qu'en dépit du fait que les adolescentes soient sexuellement actives, elles n'utilisent pratiquement pas de moyen contraceptif.

Il n'existe pas de négociation pour la conception car l'adolescente ne décide pas de sa fécondité. 
L'utilité des informations sur la SR est bien perçue par les adolescentes, mais l'expression des besoins reste très faible surtout chez les adolescentes non scolarisées. Parmi les sources d'informations citées par les adolescentes enquêtées, figurent en bonne place les cours de SVT, de puériculture dispensés dans les lycées et collèges, la radio, les séances de sensibilisation réalisées par l'Action Sociale, les services de Santé, les associations et autres projets et programmes qui interviennent dans le domaine de la SR.

Pour les adolescentes mariées, le désir d'enfant n’est pas seulement personnel, mais un besoin de conformité avec le milieu social. Ce qui justifie pourquoi leurs premières grossesses interviennent dans les mois qui suivent le mariage. Satisfaire les parents en enfantant très rapidement est le vœu de la grande majorité des adolescentes mariées.

Les résultats de l'étude indiquent que les adolescentes ont des connaissances limitées sur la grossesse hormis les signes annonciateurs de l'évènement. Elles ne disposaient pas d'informations sur la grossesse avant d'être enceintes et n’ont pas a en cherché obtenir auprès de personnes et structures ressources. Ce manque d'informations aussi bien sur la sexualité que sur la grossesse constitue l'une des principales causes de la non préparation des adolescentes pour une planification et un bon suivi de leur grossesse. Chez les adolescentes mariées ou non, ce manque d'informations est à la base des grossesses non désirées, de leur interruption volontaire, du suivi tardif et irrégulier de la grossesse.

A l'instar de la sexualité, les adolescentes perçoivent aussi l'utilité des informations sur la gestion de la grossesse, seulement les besoins sont faiblement formulés. La principale source d'informations est constituée des mères, belles-mères, sœurs, tantes ou amies ayant déjà vécu l'expérience de la grossesse.

La plupart des adolescentes interrogées n’ont pas subi de rituels ni à la connaissance de leur état de grossesse, ni pour le suivi de celle-ci. Cependant, quelques-unes d'entre elles portent des amulettes pour protéger la grossesse. Ces amulettes ont pour but de protéger la mère et l'enfant contre les «mauvais yeux », les mauvais esprits, donc d'assurer le bon déroulement de la grossesse.

Quant aux conseils et interdits liés à la grossesse, ils sont basés sur les restrictions alimentaires, vestimentaires et des comportements jugés inappropriés à leur état. Ce sont les parents qui informent les adolescentes de ces interdits dont elles ne connaissent pas la signification.

La grossesse des adolescentes est gérée par des personnes clé au niveau communautaire (la bellemère, la mère, les tantes, les maris, les AV), les agents de santé et l'Action Sociale qui s'occupent du volet socio-sanitaire. Cette gestion s'opère à partir des conseils, des actions de sensibilisation, des appuis moraux, financiers et de l'allègement des tâches de l'adolescente. Leurs interventions se situent à des niveaux différents, mais les rôles sont complémentaires.

Toutes les décisions liées à la grossesse en terme de suivi, de soins et de dépenses sont prises essentiellement par les belles-mères et les conjoints des adolescentes.

Il ressort des résultats de l'étude que les adolescentes ne recherchent pas systématiquement de l'information sur l'accouchement par crainte, mais aussi par pudeur. Elles préfèrent vivre l'expérience. On constate que l'accouchement n’est pas préparé pour la majorité d'entre elles. 
Les raisons de cette non préparation sont soit d'ordre socioculturel, soit financier. Il y a très peu de rituels liés à l'accouchement. Les rituels les plus courants sont les consultations de marabouts ou de géomanciens, l'invocation des ancêtres, les sacrifices et aumônes de toutes sortes pour faciliter l’issue de la grossesse.

Les résultats de l'étude ont montré que les itinéraires thérapeutiques des adolescentes pendant la grossesse sont soumis à plusieurs étapes, alors que l'accouchement en a une seule qui est le recours au centre de santé. La plupart d'entre elles ne vont pas systématiquement au centre de santé pour confirmer leurs grossesses, se basant sur les signes précurseurs et les dires de leurs belles-mères et mères. Elles y vont généralement tard pour la CPN, après le premier trimestre. Cependant, leur itinéraire est basé surtout sur les soins à domicile par l'automédication en cas de malaises ou de maladies quelconques. Elles se réfèrent à la médecine traditionnelle par l'utilisation de plantes et de décoctions reçues. Le centre de santé n’est que le dernier recours en cas de persistance de la maladie. Au terme de la grossesse, la presque totalité des adolescentes accouchent au centre de santé où elles reçoivent les soins adéquats donnés par les agents de santé.

La minorité qui ne fait pas de CPN a recours aux tradipraticiens et aux marabouts en cas de maladies et accouche à domicile. C'est seulement en cas de complication qu'elles sont conduites au centre de santé.

Les discussions de groupes ont montré que ces itinéraires changent lorsqu'il s'agit des adolescentes non mariées qui, voulant avorter ont recours en premier lieu à l'automédication. En cas d'échec, le deuxième recours est le tradipraticien qui leur donne des produits. Le centre de santé est le dernier recours après plusieurs tentatives. Elles restent dans la " clandestinité » avec leurs grossesses et très peu d'entre elles repartent au centre de santé pour faire la CPN. Certaines d'entre elles arrivent à avorter, d'autres non. Ces dernières font parfois appel à l'Action Sociale en cas de problème de paternité. Quelques unes accouchent au CSPS et les autres à domicile. A cause de la situation complexe dans laquelle la grossesse des adolescentes non mariées survient, il est difficile de tracer un itinéraire thérapeutique très précis de cette cible qui se cache du fait de la honte qu’elles éprouvent vis-à-vis de leur état.

L'étude a révélé que les premiers soins reçus par les adolescentes post-partum et leurs nouveaunés sont donnés par les agents de santé à la maternité. Par la suite, ils reçoivent d'autres soins à base de plantes médicinales à domicile. Ces soins sont généralement prodigués par les bellesmères, mères ou autres femmes de la famille dans le but de rendre la mère et le bébé bien portant.

Il est ressorti que la grande majorité des adolescentes post-partum ont une méconnaissance des signes qui doivent alerter sur la santé de la mère ou de l'enfant. Dans les deux cas, leur premier recours en cas de maladie est l'automédication par les décoctions. Ensuite, elles se rendent dans une formation sanitaire si leur état de santé ne s'améliore pas. Parfois, une association des soins traditionnels et modernes est faite en même temps. Cependant, les résultats de l'étude montrent que pour certains types de services tels que les vaccins, les consultations, la planification familiale, les adolescentes ont toujours recours au centre de santé.

Hormis la maternité, les soins à domicile, l’itinéraire des adolescentes post-partum se poursuit aussi au niveau des guérisseurs traditionnels qui les soignent pour des maux de ventre, des ballonnements, et aussi pour avoir du lait. En plus de l'adolescente, les guérisseurs s'occupent de 
la santé des nourrissons ou se chargent de donner des conseils aux parturientes sur leur entretien, leur alimentation et leur hygiène.

Les adolescentes, dans leur majorité, disposent de peu d'informations en matière de SR et sont disposées à en avoir.

L'étude a fait ressortir l'existence de difficultés au niveau des structures de prise en charge des adolescentes telles que l'Action Sociale et la Santé. Ces dernières ont formulé leurs besoins en terme de renforcement de compétences, de capacités institutionnelles et matérielles. 
La population burkinabé est majoritairement jeune, les personnes de moins de 20 ans représentent $57,0 \%$ de la population ${ }^{1}$. Cette situation est particulièrement préoccupante, tant du point de vue sanitaire, éducatif, économique que social. En effet, depuis quelques années, les enjeux liés à la sexualité, à la fécondité et à la santé reproductive, ainsi que les problèmes y afférents, ont fait l'objet d'une attention soutenue au niveau du pays. Ces adolescent(e) $\mathrm{s}^{2}$, qui constituent $22,98 \%$ de cette population sont confrontés à de nombreux risques tels que les relations sexuelles précoces, les grossesses non désirées, les mariages précoces, la propagation des IST, le Sida et les mutilations génitales féminines. Ces problèmes sont vécus différemment suivant le sexe, l'environnement géographique, économique ainsi que socioculturel. Compte tenu du poids démographique de cette population jeune, de son exposition à la pauvreté et notamment du faible accès aux services sociaux de base, l'Etat s'investit depuis plusieurs années, en collaboration avec des partenaires au développement dans la prise en charge des problèmes de santé sexuelle et reproductive des adolescents liés aux grossesses et mariages précoces.

En effet, le mariage précoce des adolescentes constitue un fait majeur au Burkina Faso, spécifiquement dans les zones rurales. Dès l'âge de 17 ans, une jeune fille sur cinq (21\%) a déjà commencé sa vie féconde et, à 19 ans, cette proportion est de $57 \%$ dont la grande majorité (48 \%) a déjà, au moins un enfant. D’après les résultats d'une étude menée conjointement par l'INSD et UNICEF en 2008, les femmes âgées de 15-19 ans, 23,5\% sont déjà mariées ou vivent en unions libres dont 30,9\% d'entre elles sont en milieu rural contre 9,5\% en milieu urbain. Cette situation a pour corollaire une fécondité précoce élevée. Selon les données de l'EDS ${ }^{3}$, à 15 ans, $10 \%$ des adolescentes sont en union et l'accouchement survient en général dans les 20 premiers mois suivant le mariage. A $15-19$ ans, cette fécondité est à $131 \%{ }^{4}$. On constate que près d'un quart des jeunes femmes de 15-19 ans (23\%) ont déjà commencé leur vie féconde : $17 \%$ ont déjà, au moins un enfant et 6 \% sont enceintes pour la première fois

Les risques associés au premier accouchement sont élevés au Burkina Faso parce que la majorité des accouchements se font sans une assistance médicale et à domicile. En effet, seulement $57,26 \%{ }^{5}$ des accouchements sont assistés. Chez les moins de 20 ans, ce fait représente $64 \%$ des accouchements sans assistance médicale ${ }^{6}$. Ces risques qui se résument entre autres au décès de l'enfant et/ou de la mère découlent du déficit d'informations sur la santé sexuelle et reproductive des adolescentes.

Une meilleure prise en charge des conséquences liées aux mariages et grossesses précoces doit non seulement passer par une amélioration du niveau de connaissances des adolescent(e)s, mais surtout de celui des parents et des personnes ressources sur les questions relatives à la santé de la reproduction. Il découle de cette situation de nouvelles attitudes et un changement qualitatif de

\footnotetext{
${ }^{1}$ RGPH (résultats définitifs) 2006

${ }^{2}$ Dans le cadre de cette étude, est prise en compte les adolescentes de 10 à 19 ans.

${ }^{3}$ EDS 98/99

${ }^{4}$ EDS 2003

${ }^{5}$ Annuaire statistique 2007

${ }^{6}$ EDS 98/99
} 
comportement de ceux qui sont chargés de l'éducation et de la prise en charge de ces adolescentes tant au niveau familial que communautaire.

Plusieurs études ont été menées dans le domaine de la santé reproductive des adolescent(e)s, mais une étude complémentaire sur « l'itinéraire thérapeutique des adolescentes en grossesse et postpartum » s'avère nécessaire. En effet, l'environnement socioculturel des adolescent(e)s et les repères sociaux en matière d'éducation-conseil sont censés leur donner les moyens nécessaires pour une meilleure compréhension et une prise en charge des questions relatives à la sexualité, à la grossesse et à l'accouchement.

Le Projet «Eliminer le mariage des enfants au Burkina: un plan pour la protection, le renforcement et l'action communautaire» vise à renforcer les compétences de vie des adolescentes pour leur permettre d'avoir une maîtrise sur leurs droits au développement et à la santé de la reproduction. Cela suppose d'une part, qu'on leur fournisse des informations, de l'éducation et d'autre part qu'on leur offre des services en matière de santé sexuelle et reproductive de qualité destinés à les protéger contre les conséquences négatives d’une sexualité non contrôlée.

Dans le but d'orienter les stratégies et activités de ce projet, il était important de faire un diagnostic de l'itinéraire thérapeutique des adolescentes pendant la grossesse, pour l'accouchement et la période post-partum en vue de mieux cerner leurs connaissances, attitudes, pratiques et perceptions dans le domaine de la SR. D’où la présente recherche qui permettra de rendre disponible le maximum d'informations permettant de développer des stratégies pour une amélioration de la gestion des grossesses, de l'accès, de la fréquentation des centres de santé ainsi que de la qualité des soins offerts.

\section{OBJECTIFS ET RESULTATS ATTENDUS}

\subsection{Objectif général}

Etudier les itinéraires thérapeutiques des adolescentes en grossesse et post-partum en vue d'accroître la fréquentation des centres de santé et surtout le nombre des accouchements assistés.

\subsection{Objectifs spécifiques}

Comme objectifs spécifiques, il s’agit de :

* Faire un état des lieux sur les connaissances, perceptions, attitudes et pratiques en matière de grossesse et d'accouchement des adolescentes mariées ou non dans les zones d'intervention ;

* Recueillir des informations sur le vécu et les trajectoires de grossesse, d'accouchement et de post-partum des adolescentes ;

* Evaluer les différents besoins des adolescentes en terme d'accès aux centres de santé, à la qualité des soins et aux informations sur la santé de la reproduction ;

* Évaluer le niveau de connaissance des personnes ressources sur les questions relatives à la grossesse, au suivi de la grossesse, à l'accouchement, au post-partum et au suivi des nourrissons ;

* Identifier les types de collaboration entre les personnes ressources et les acteurs qui interviennent dans la gestion de la grossesse, de l'accouchement et de la période postpartum des adolescentes ; 
Evaluer les offres de prestation des services socio-sanitaires en vue de faire des propositions qui contribueront à accroître la fréquentation des centres de santé et le nombre des accouchements assistés dans la zone d'intervention du projet.

\subsection{Résultats attendus}

A la fin de l'étude, les résultats suivants sont attendus:

* l'état des lieux sur les connaissances, perceptions, attitudes et pratiques en matière de grossesse et d'accouchement des adolescentes mariées ou non dans les zones d'intervention est connu ;

* des informations sur le vécu et les trajectoires de grossesse, d'accouchement et de la période post-partum des adolescentes sont disponibles ;

* les différents besoins des adolescentes en terme d'accès aux centres de santé, à la qualité des soins et d'informations sur la santé de la reproduction sont recensés ;

* le niveau de connaissance des personnes ressources sur les questions relatives à la grossesse, au suivi de la grossesse, à l'accouchement, au post-partum et au suivi des nourrissons est évalué ;

* les types de collaboration entre les personnes ressources et acteurs qui interviennent dans la gestion de la grossesse et de l'accouchement des adolescentes sont identifiés ;

* des interventions qui contribueront à accroître la fréquentation des centres de santé et le nombre des accouchements assistés dans la zone d'intervention du projet sont proposées.

\section{DEMARCHE METHODOLOGIQUE}

D’une façon générale, la démarche adoptée a été la suivante :

* Une revue de la littérature a été effectuée à partir des documents de base du projet et d'autres ouvrages et études relatifs au sujet. L'objectif a été de faire le point des actions réalisées à partir des documents, de disposer d'informations pour orienter les différents axes de l'étude. Les informations collectées à partir des documents et celles collectées sur le terrain sont complémentaires.

La collecte des données sur le terrain a été réalisée sous forme d'entretiens semistructurés. Cette étape de la recherche s’est effectuée en deux phases :

- La première phase a consisté à organiser et à approfondir les thèmes mis en évidence à l'issue des entretiens semi-structurés ;

- La seconde phase a permis de réaliser des focus groups.

Les entretiens ont été effectués auprès des différents acteurs impliqués dans la gestion de la grossesse des adolescentes (les adolescentes elles-mêmes, les personnes ressources, les guérisseurs, les agents de santé). 
Une comparaison des attitudes, comportements et pratiques a été faite également entre les expériences selon les zones d'études en vue de faire ressortir les ressemblances et les points spécifiques. En outre, des récits de vie pathétiques ont été recensés pour illustrer la problématique de l'adolescente en grossesse ou post-partum.

\subsection{Zones d'intervention et sites de l'étude}

Le principe n'a pas été de prendre en compte les 24 localités d'intervention du projet qui sont réparties dans les cinq régions: le Centre-Sud, l’Est, le Sahel, le Centre-Est et le Centre-Nord, mais de se limiter à 12 .

La collecte des données a été faite sur un échantillon assez représentatif de sites. Un choix raisonné basé sur des critères pertinents des zones de collecte a été opéré en tenant compte des éléments suivants:

- l'ampleur du sujet d'étude dans les localités pour donner une vue d'ensemble du phénomène dans tous ses contours ;

- $\quad$ un site urbain versus site rural.

Les sites (Gaongo, Nafbanka, Natiaboani et Koaré) ayant été déjà explorés lors de l'étude sur « les itinéraires thérapeutiques des adolescentes en grossesse et $1^{\text {ere }}$ fois parents au Bazèga et au Gourma en 2003 » n’ont pas été retenus.

Tableau $N^{\circ} 1$ : Répartition des sites de l'étude selon les régions

\begin{tabular}{|l|l|l|l|l|}
\hline \multicolumn{1}{|c|}{$\begin{array}{c}\text { Région du } \\
\text { Centre Sud }\end{array}$} & Région de l'Est & \multicolumn{1}{|c|}{$\begin{array}{c}\text { Région du } \\
\text { Sahel }\end{array}$} & \multicolumn{1}{|c|}{$\begin{array}{c}\text { Région du } \\
\text { Centre Est }\end{array}$} & Centre Nord \\
\hline 1. Manga & 3. Bogandé & 5. Dori & 8. Tenkodogo & 11. Kaya \\
\hline 2. Gogo & 4. Manni & 6. Saouga & 9. Ouargaye & 12. Noaka \\
\hline & & 7. Tibilindi & $\begin{array}{l}\text { 10. Département } \\
\text { de Bissiga }\end{array}$ & \\
\hline
\end{tabular}

\subsection{Population cible de l'étude}

Les critères d’âge, les situations matrimoniales (mariée ou célibataire) ont guidé dans le choix des adolescentes.

L'étude a été menée auprès d'un échantillon de tous les groupes cibles et acteurs impliqués dans la gestion de la grossesse de l'adolescente, de l'accouchement, des soins au nouveau-né. Ce sont :

- les adolescentes mariées en grossesse de 10 à 19 ans ;

- les adolescentes mariées post-partum de 10 à 19 ans ;

- les adolescentes non mariées en grossesse de 10 à 19 ans ;

- les adolescentes non mariées post-partum de 10 à 19 ans ;

- les adolescentes célibataires n’ayant pas vécu d'expériences de la grossesse de 10 à 19 ans

- Les maris des adolescentes en grossesse et post-partum. 
Les personnes ressources et structures impliquées dans la gestion de la grossesse et de l'accouchement sont :

- les belles-mères;

- les accoucheuses villageoises ;

- les guérisseurs ;

- les agents de santé ;

- l'Action Sociale ;

- les ONG et associations prestataires de services.

D’autres cibles qui interviennent indirectement dans la gestion de la grossesse ont été également rencontrées. Il s’agit des responsables religieux et les chefs traditionnels.

\subsection{Collecte de données sur le terrain}

Des entretiens individuels approfondis et des focus-group ont été utilisés pour la collecte des données à l'aide de guides et de questionnaires.

La collecte des données a duré 30 jours. Elle s’est déroulée du 21 octobre au 20 novembre 2008. Elle a concerné les douze sites retenus dans le cadre de cette étude.

Dans chaque localité, l'équipe des consultants a travaillé en étroite collaboration avec des guides locaux qui l'ont aidée à identifier les cibles concernées par la recherche et à mobiliser la population pour les entretiens individuels et les focus-groups.

La collecte a été faite par région selon le plan de route élaboré par l'équipe. L’itinéraire du plan a été le suivant : le Sahel, le Centre-Nord, le Centre-Est, l'Est et le Centre-Sud : 2 ou 3 sites ont été retenus par région. Avant l'arrivée de l'équipe sur le terrain, des contacts ont été pris en vue de faciliter la collecte des données.

Au niveau de chaque site, pour les entretiens individuels, les cibles suivantes ont été rencontrées:

- les adolescentes mariées en grossesse et post-partum ;

- les adolescentes non mariées en grossesse et post-partum ;

- les adolescentes célibataires n'ayant pas vécu d'expérience de la grossesse ;

- Les maris des adolescentes en grossesse et post-partum ;

- les belles-mères;

- les accoucheuses villageoises ;

- les guérisseurs ;

- les agents de santé ;

- l'Action Sociale ;

- les ONG et associations prestataires de services ;

Les focus-group ont été réalisés auprès des cibles suivantes :

- les adolescentes en grossesse et post-partum ;

- les adolescentes célibataires n’ayant pas vécu d'expériences de la grossesse.

\subsection{Traitement et analyse des données}


Les données qualitatives ont été traitées manuellement. Cependant, pour l'analyse, une triangulation des informations recueillies dans les différentes phases de la collecte a été privilégiée.

\subsection{Difficultés et limites de l'étude}

\subsubsection{Difficultés}

Comme difficultés de terrain, on note :

- La période de collecte des données (octobre - novembre) ayant coïncidé avec les récoltes dans les champs, a été peu favorable à l'administration des guides d'entretiens à cause de la non disponibilité de certaines cibles tels les maris et les adolescentes ;

- La difficulté de rencontrer les adolescentes en grossesse dans les 5 régions car elles éprouvaient de la honte sous le regard des autres ; surtout les adolescentes non mariées.

\subsubsection{Limites}

- La sensibilité du sujet n'a pas permis d'avoir des réponses à certaines questions surtout sur la sexualité quelle que soit la région, limitant ainsi la possibilité de prospection dans la recherche d'informations ; 


\section{RESULTATS DE L'ETUDE}

\subsection{Caractéristiques sociodémographiques des enquêtées}

Au total, 222 entretiens individuels ont été réalisés sur 296 prévus et 17 focus-group sur 20 prévus. Cela s'explique par la difficulté de mobilisation de certaines cibles telles que les bellesmères et les adolescentes en grossesse et celles post-partum.

Le résumé des entretiens individuels avec les différentes cibles donne les résultats suivants : 52 dans la Région du Sahel, 39 au Centre-Nord, 62 au Centre-Est, 39 à l'Est et enfin 30 au CentreSud.

\section{Tableau $\mathrm{N}^{\circ} 3$ : Nombre d'entretiens individuels réalisés par région}

\begin{tabular}{|c|c|c|c|c|c|c|c|}
\hline $\mathbf{N}^{\circ}$ & Entretiens individuels & Sahel & Centre-Nord & Centre-Est & Est & Centre-Sud & Total \\
\hline 1 & Adolescentes en grossesse mariées & 6 & 4 & 8 & 4 & 2 & 24 \\
\hline 2 & Adolescentes en grossesse non mariées & 2 & 3 & 2 & 2 & 5 & 14 \\
\hline 3 & Adolescentes post-partum mariées & 6 & 3 & 8 & 3 & 1 & 21 \\
\hline 4 & Adolescentes post-partum non mariées & 3 & 3 & 1 & 3 & 4 & 14 \\
\hline 5 & Maris adolescentes en grossesse et post-partum & 8 & 6 & 9 & 6 & 4 & 33 \\
\hline 6 & $\begin{array}{l}\text { Belles-mères adolescentes en grossesse et post- } \\
\text { partum }\end{array}$ & 9 & 5 & 8 & 6 & 4 & 32 \\
\hline 7 & Accoucheuses villageoises & 3 & 1 & 1 & 0 & 1 & 6 \\
\hline 8 & Guérisseurs & 3 & 1 & 3 & 2 & 0 & 9 \\
\hline 9 & Leaders religieux & 4 & 5 & 8 & 6 & 5 & 28 \\
\hline 10 & Chefs coutumiers & 2 & 2 & 3 & 2 & 1 & 10 \\
\hline 11 & Responsables administratifs & 2 & 1 & 3 & 0 & 0 & 6 \\
\hline 12 & Action Sociale & 2 & 1 & 2 & 2 & 1 & 8 \\
\hline 13 & Santé & 2 & 2 & 3 & 2 & 2 & 11 \\
\hline \multirow[t]{2}{*}{14} & ONG et Associations prestataires & 0 & 2 & 3 & 1 & 0 & 6 \\
\hline & Total & 52 & 39 & 62 & 39 & 30 & 222 \\
\hline
\end{tabular}

\subsubsection{Adolescentes en grossesse}

Au total, 38 adolescentes en grossesse ont été interviewées dans les 12 sites de l'étude parmi lesquelles on distingue 24 mariées (dont 1 dans un ménage polygame, 23 dans des ménages monogames) et 14 célibataires.

Leur âge moyen au moment de l'enquête est de 17,6 ans, l'âge moyen au mariage est de 16,4 ans. L'âge le plus bas au mariage est de 13 ans.

Quant au groupe ethnique, on a pu dénombrer 25 Mossi, 6 Peulhs, 5 Gourmantché, 1 Bobo et 1 Gourounsi.

Le niveau d'instruction fait ressortir que 9 adolescentes sont sans niveau scolaire, 11 ont un niveau primaire, 10 ont un niveau secondaire et 8 sont alphabétisées en langues nationales.

La durée moyenne du mariage est de 1 an et demi et l'âge moyen de la grossesse est de 6 mois. La majorité des adolescentes est chrétienne (23) suivi des musulmanes (15). 


\subsubsection{Adolescentes post-partum}

En résumé, 35 adolescentes post-partum ont été rencontrées dans le cadre de la collecte des données. Elles ont une moyenne d'âge de 17,3 ans, avec un âge moyen au mariage de 15,4 ans et 12,5 ans comme âge le plus bas au mariage. La situation matrimoniale fait ressortir que : 18 femmes sont dans des ménages monogames (il s’agit pour la plupart d’unions libres et de mariages traditionnels.) ; 3 femmes sont en situation de polygamie et 14 sont des célibataires.

12 des adolescentes post-partum interviewées sont sans niveau scolaire contre 6 de niveau primaire, 14 de niveau secondaire et 3 alphabétisées en langues nationales.

Quant à leur groupe ethnique, on dénombre 10 femmes Peulh, 20 Mossi, 4 Gourmantchés et 1 Bissa. Les adolescentes appartiennent à deux religions que sont le christianisme (11) et l'islam (24).

\subsubsection{Belles-mères}

Ce sont 32 belles-mères au total qui ont été rencontrées dans les 12 sites de l'étude avec une moyenne d'âge de 49,6 ans, une moyenne d'âge au mariage de 17,2 ans et 35,7 ans comme durée moyenne de mariage. Parmi elles, 17 sont en ménages monogames et 15 en ménages polygames.

Le niveau d'instruction montre que 20 femmes sont sans niveau scolaire, 6 ont un niveau primaire, 2 sont du secondaire et 4 sont alphabétisées en langues nationales.

Ainsi, on dénombre 16 femmes Mossi, 9 Peulhs, 6 Gourmantchés et 1 Bobo. Au plan religieux, elles sont 17 chrétiennes, 12 musulmanes, 3 animistes.

\subsubsection{Maris d'adolescentes}

Au total, 33 maris d'adolescentes ont été interviewés dans le cadre de cette étude parmi lesquels on distingue : 14 maris d'adolescentes en grossesse et 19 maris d'adolescentes post-partum.

Leur âge moyen est de 25,5 ans. L'âge moyen au mariage des maris d'adolescentes en grossesse est de 18 ans contre 22,8 ans pour les maris d'adolescentes post-partum. L'âge le plus bas des maris est de 17 ans et le plus élevé 50 ans. On dénombre 31 monogames et seulement 2 polygames. 5 maris sont sans niveau scolaire, tandis que 15 ont un niveau primaire, 9 un niveau secondaire et 4 alphabétisés en langues nationales.

Ils se repartissent dans 4 groupes ethniques à savoir: les Peulh (8), les Mossi (18), les Gourmantchés (6) et Bissa (1). Sur le plan religieux ils sont 16 Chrétiens et 17 Musulmans. 


\subsubsection{Guérisseurs}

Sur les neuf (09) guérisseurs enquêtés, quatre (04) sont des femmes. Les guérisseurs sont des personnes âgées de plus de 43 ans. Ils appartiennent aux groupes ethniques mossi, peulh et gourmantché. Dans l'ensemble, ils ont en moyenne plus de 26 ans d'expériences dans l'exercice de leur métier.

\subsubsection{Accoucheuses villageoises (AV)}

Sur les 6 AV rencontrées, 3 sont mossi et 3 peulhs. Leur âge moyen est de 45,5 ans avec au minimum 16,7 ans d'exercice du métier. Parmi elles, 5 sont des familles polygames et 1 est célibataire ; 4 sont musulmanes et 2 chrétiennes. Le niveau scolaire laisse paraître 1 sans niveau, 1 avec un niveau primaire, 2 avec un niveau secondaire et 2 alphabétisées en langues nationales.

En plus des entretiens individuels, des focus-group ont été réalisés dans les sites de l'étude. Au total, 17 focus sur 20 prévus ont été réalisés. Ils ont concerné les adolescentes, les adolescentes en grossesse et post-partum.

4 focus-group ont été réalisés dans le Sahel, 3 au Centre-Nord, 5 au Centre-Est, 3 à l'Est et enfin 2 au Centre-Sud.

Le tableau ci-dessous fait le récapitulatif des focus par région:

Tableau $\mathrm{N}^{\circ} 4$ : Nombre de focus-group réalisés par région

\begin{tabular}{|l|c|c|c|c|c|c|}
\cline { 2 - 7 } \multicolumn{1}{c|}{} & Sahel & Centre-Nord & Centre-Est & Est & Centre-Sud & Total \\
\hline Focus ados & 2 & 2 & 3 & 2 & 2 & $\mathbf{1 1}$ \\
\hline $\begin{array}{l}\text { Focus adolescentes en } \\
\text { grossesse et post-partum }\end{array}$ & 2 & 1 & & & & \\
\hline Total & $\mathbf{4}$ & $\mathbf{3}$ & $\mathbf{5}$ & $\mathbf{3}$ & $\mathbf{2}$ & $\mathbf{1 7}$ \\
\hline
\end{tabular}

\subsection{Sexualité et représentation sociale de la maternité dans les zones de l'étude}

Selon une étude de Family Care International/Burkina Faso et Niger intitulée, «Vous, votre vie, vos rêves : bien réussir votre adolescence ", la sexualité est complexe et va au-delà des désirs ou rapports sexuels. Elle comprend :

- la conscience et les sentiments à propos du corps de l'adolescent et du corps des autres ;

- la capacité et le besoin de l'adolescent d'être proche d'un point de vue affectif de quelqu'un d'autre ;

- la compréhension de ce que signifie être femme ou homme ;

- l'attraction sexuelle envers d'autres personnes ;

- la capacité physique en termes de reproduction.

L'étude souligne également que la sexualité est une partie importante, agréable et naturelle de la vie. Mais, parfois, les gens l'utilisent de manière malsaine pour influencer ou contrôler d'autres personnes. Ceci peut aller du flirt et de la séduction au harcèlement et à l'abus sexuel. La notion de la sexualité n'est pas bien perçue par les adolescents et adolescentes. L'adolescence constitue 
une période de naïveté et d'insouciance qui les expose aux problèmes de santé sexuelle et reproductive dont les grossesses non désirées et/ou précoces.

Si la maternité précoce est inscrite dans l'histoire des femmes depuis toujours, de nos jours, elle a atteint des proportions inquiétantes.

\subsubsection{Perceptions de la procréation/maternité}

Sur le plan anthropologique, la maternité est un phénomène social valorisant dans presque toutes les sociétés. En effet, elle assure non seulement la pérennisation de la lignée familiale mais aussi valorise la femme. La naissance d'un enfant dans une famille est un événement capital, un moment d'espoir pour tous. La procréation revêt un caractère noble et de ce fait doit obéir aux normes sociales c'est-à-dire se dérouler dans le cadre strict du mariage.

D’après Ida Christiane TAMINI (Janvier 1999), la procréation n'est pas seulement un phénomène biologique, elle est également un phénomène social valorisant. Dans la société traditionnelle, une femme qui enfante est une femme qui a pleinement rempli son contrat social. La procréation occupe pour ainsi dire une place de choix, ce qui justifie la représentation de la fécondité à travers des masques, des statuettes, des dieux et même des rituels.

Si l'homme n'a besoin que du mariage pour être admis comme un adulte, la femme doit attendre d'avoir procréer pour se voir reconnaître un certain degré de maturité psychologique. Son intégration sociale en dépend. Ce n'est qu'à ce moment qu'elle deviendra un maillon capital dans la chaîne des vivants et des morts. C'est l'enfant qui lui permettra de prendre place dans la maison de son mari et dans le lignage de ce dernier.

L'aptitude à la procréation est assujettie à un certain nombre de critères spécifiques aux hommes et aux femmes.

L'apparition des menstruations constitue pour la plupart des personnes ressources interrogées le principal avertisseur qui témoigne de la capacité de procréation d’une fille et annonce aussi le moment du mariage.

En plus des règles, certains font état du développement physique qui se caractérise par le développement des seins, du bassin, de l'ossature, etc. A l'unanimité, hommes et femmes s'accordent que l'apparition des menstrues est plus déterminante que la forte corpulence quant à l'aptitude de la fille à procréer.

La détermination de l'âge auquel la fille est apte à procréer est en relation avec l'appartenance religieuse, ethnique et souvent même à la zone géographique :

- Au Sahel où dominent l'ethnie peulh et l'Islam, la capacité à procréer n’est pas liée à l'âge, mais à l'apparition des règles et au développement physique de la fille. les filles sont mariées entre 10 et 15 ans. Le mariage précoce y est une pratique dominante, quel que soit le niveau d'instruction et la catégorie socioprofessionnelle des parents. Sa pratique est une forme de prévention contre les grossesses non désirées.

Les filles se marient ici généralement à partir de 10 ans et les garçons à partir de 13 ans. Les filles ont leur première grossesse à partir de 15 ans » (Imam, 78 ans, Malébé, Saouga). 
"Ici, à partir de 8 ans, si tu n’as pas de prétendant, tu n’es pas considérée. » [Accoucheuse auxiliaire, CSPS Saouga].

"Ici, l'âge idéal au mariage pour les filles est de 15 ans. Cet âge a été dicté par l'Islam qui dit qu'une fille de 15 ans est assez mûre pour désigner la personne qu'elle aime. »(Chef de village, 80 ans, musulman, Saouga).

- Au Centre-Nord où dominent les Mossi et les musulmans, la capacité à procréer est liée aux âges impairs de 17, 19, 21 ans. Selon leurs croyances, les chiffres pairs ne portent pas bonheur. Aussi, une fille qui n'a pas pu se marier à 17 ans doit attendre 19 ou 21 ans, même si elle a un prétendant. Cette pratique du mariage se retrouve également au CentreSud. Elle a une répercussion sur l'âge de la maternité qui est un peu plus retardé qu'au Sahel.

"Ici, on destine les filles en mariage en bas âge, à 10 ans. Cet acte est appelé "Siourê » («destiné à » en mooré). Mais, le mariage n'est célébré que lorsque la fille atteint les 17 ans » (Naba Koom, Chef de Kaya).

"Si quelqu'un voit une fille qu'il veut et qu'elle aussi le veut, nous lui disons d'attendre qu'elle ait 17 ou 19 ans. Ici, à Noaka, le mariage est interdit avant 17 ans. Il y a des âges où la fille est donnée en mariage : 17, 19, 21 ou 23 ans. Mais, il peut arriver qu'une fille soit mariée avant ces âges. Cela se fait seulement à 15 ans lorsque c'est une fille qui pose problèmes ou qui est tombée enceinte. » (Imam, 60 ans, mossi, /Noaka).

«Selon les coutumes, les filles se marient à l'âge de 19-21 ans, car c'est à cet âge que le mariage est béni. » (Pasteur, 46 ans, mossi, /Gogo).

Il ressort donc de ces citations qu'au Centre-Nord, on pratique aussi le don de fille en bas-âge, mais le mariage n’est célébré généralement qu’à partir de 17 ans. Cette pratique est une forme de prévention contre les grossesses non désirées surtout pour les filles non scolarisées.

Quant à celles qui sont en cours de scolarisation, elles échappent «au don de femme » et sont libres de se marier à l'âge voulu et de choisir leurs conjoints.

- Au Centre-Est, la capacité à procréer est déterminée aussi par l’âge de 17 ans qui correspond à l’âge au mariage dans la localité.

Cependant, on note l'existence de deux spécificités que sont le rapt et l'échange de fille appelé "Lito » en «Zaonré ». Ce sont des pratiques culturelles fortement ancrées qui sont toujours en vigueur dans la localité.

Le rapt consiste à l'enlèvement de jeune fille avec ou sans le consentement de celle-ci et de ses parents. Le rapt, selon le Président du tribunal départemental de Bissiga, est pratiqué surtout sur les adolescentes de 13 à 16 ans et a lieu généralement en fin d’hivernage. 
"Ici, les garçons enlèvent les filles dans les marchés. Si la famille donne une fille en mariage et qu'elle refuse, les parents l'autorisent à l'enlever» (Accoucheuse auxiliaire, Bissiga).

«Je reçois beaucoup de plaintes de parents pour cause de rapt de leurs filles : exemple de 2 élèves de CM2 enlevés par deux garçons. Une des 2 a pu s'échapper et j'ai dû intervenir pour récupérer la $2^{\text {ème }}$ et la remettre à sa famille; rien qu'en juin dernier, je suis également intervenu pour retirer une autre fille enlevée... » (Préfet/Président du tribunal départemental de Bissiga).

L'échange de fille est un arrangement entre deux familles amies, parents. Cette pratique exige que la famille réceptrice donne en retour une fille. Mais, si elle n'en dispose pas, l'autre famille est obligée d'attendre qu'une fille soit disponible, sinon on reprend la fille. Cette pratique concerne les filles de tous les âges, même celles qui ne sont pas encore nées. Cela est rencontré surtout dans la localité de Bissiga et de Ouargaye.

Bien qu'elles soient culturelles, ces pratiques impliquent des conséquences souvent difficiles à gérer par les familles et les autorités locales. Au-delà des mésententes qu'elles peuvent générer entre les familles, des problèmes de paternité et, de déscolarisation des filles, le plus grave, est l'exposition des adolescentes à des risques liés aux grossesses non désirées et/ou précoces.

«Nous pratiquons ici l'échange de fille. Si je te donne une fille et que tu n'en as pas pour me donner en retour, si elle accouche elle va rembourser. Cela concerne surtout les filles âgées de 17 et 18 ans. Si on les donne avant cet âge, elles vont souffrir» (Chef coutumier, 78 ans, Bissiga).

"Il existe ici la pratique de l'échange de filles entre famille. Lorsqu'un homme veut une fille en mariage, il doit proposer ou promettre sa sœur en retour sinon il ne peut pas l'épouser. Les âges indiqués sont de 15 à 17 ans » (Préfet/Président du tribunal départemental de Ouargaye).

- A l'Est, il n'y a pas d'âge fixe pour le mariage de la fille. Selon les personnes ressources enquêtées, cet âge varie de 13 à 17 ans. A part quelques exceptions, où certaines adolescentes jugées «indomptables » par les parents sont données très tôt en mariage pour éviter qu'elles tombent enceintes, (ce qui constitue un déshonneur pour la famille) l'âge du mariage est retardé par les parents qui veulent profiter le plus longtemps possible des services domestiques assurés par leurs filles.

"Il n'y a pas d'âge fixe pour le mariage ; mais généralement c'est situé entre 17 et 19 ans. Pour celles qui sont pressées, on les donne à 15 ans... Des fois, le mariage dépend aussi de la corpulence de la fille. » (Chef de canton, 80 ans, Gourmantché, Animiste/Bogandé).

"Le mariage des filles à 13-14 ans est un cas exceptionnel. Le milieu familial ne se trompe pas car les parents observent les agissements de la fille avant de décider de la marier à cet âge. Si elle s'agite beaucoup, il faut la donner tôt en mariage pour ne pas avoir des surprises désagréables. » (Abbé, 60 ans, Mossi/Bogandé).

«Avant, nous donnions nos filles en mariage à 17 ans mais maintenant, c'est contre notre gré ; Nos filles prennent les murs les nuits, se prostituent et nous amènent des grossesses non désirées. 
C'est pour cette raison que nous préférons les marier très tôt... Lorsque la croissance de la fille est bien, à partir de l'apparition de ses règles, on peut la donner en mariage à 14-15 ans » (Imam de la grande mosquée, 37 ans, Gourmantché/Manni).

A l'instar du Centre-Est, le rapt appelé aussi « bitchono » en Gulmancema est aussi pratiqué à l'Est. De plus, le mariage forcé est aussi une pratique de la localité et concerne surtout les fillettes âgées de 11 à 12 ans. Même si la fille refuse, elle est forcée malgré elle à consommer le mariage avec tous les risques qu'elle peut encourir. Il existe aussi des cas de dons de filles en bas-âge; dans ce cas, la fille reste chez ses parents jusqu'à l'âge de 14-15 ans voire plus si elle se comporte bien. Quelque soit la forme de mariage pratiquée, la survenue de grossesses précoces et/ou non désirées est inéluctable. Cela expose les adolescentes à des problèmes de SR tels que: les avortements spontanés et accouchements prématurés ou difficiles avec des taux de césariennes élevés ; les déchirures du périnée et autres complications telles que les fistules et infections génitales pouvant aboutir, souvent, à une stérilité, le traumatisme du nouveau-né et le faible poids à la naissance.

De plus, le cancer du col de l'utérus est plus fréquent chez les femmes qui ont commencé leur activité sexuelle (ou ont été mariées) avant l’âge de 17 ans.

«Une fille de 13-14 ans a été enlevée par un monsieur qui l'a violée. Cet acte a traumatisé la fille et elle est sur le point de devenir folle. Elle a été envoyée dans un centre pour soins » (Action Sociale, Bogandé).

"J'ai été témoin du mariage forcé d'une adolescente de 17 ans. Les parents ont dit que le prétendant a trop travaillé dans leur champ (5 à 6 ans). Mais, la fille avait un copain avec qui elle projetait de se marier. Les parents ont dit qu'elle était obligée de se marier au prétendant de leur choix. » (Action Sociale, Manni).

Au regard des informations collectées, il ressort que l'Est est un foyer qui réunit toutes les formes de mariages précités qui favorisent la sexualité précoce. La présence de toutes ces pratiques dans cette localité mérite qu'une attention accrue lui soit accordée car l'ensemble de ces pratiques influencent négativement sur la maternité et la SR des adolescentes.

Pour les hommes, outre le critère de l'âge, les critères appliqués pour définir leur aptitude à procréer sont essentiellement d'ordre social et économique. La détermination de l’âge idéal pour un homme pour avoir un enfant est fonction de son statut socio-économique comme l'affirment ces maris d'adolescentes :

" Je souhaitais avoir un enfant à 20 ans, mais compte tenu de ma situation économique, j'ai préféré attendre d'avoir 28 ans » (Mari d'adolescente en grossesse, 28 ans, Mossi, Catholique/Noaka).

« J'ai eu mon $1^{\text {er }}$ enfant à 18 ans, j'aurais voulu attendre d'avoir 25 ans car je voulais planifier d'abord ma vie, avoir mon propre boulot. Je regrette un peu car je n'ai pas l'âge de gérer une famille. » (Mari d'adolescente post-partum, 19 ans, Mossi, Catholique/Manga).

$\mathrm{Si}$, selon les valeurs et normes sociales traditionnelles, l'enfantement est un acte valorisant qui doit survenir dans le cadre strict du mariage, de nos jours, avoir un enfant sans être marié est de 
plus en plus toléré. Il en découle une augmentation du nombre d'adolescentes mères célibataires mais aussi d'adolescentes mariées précocement due aux tentations et pressions dont sont victimes les filles.

\subsubsection{Sexualité chez les adolescentes}

Le contenu de la revue littéraire a fait ressortir une sexualité très active chez les adolescents et adolescentes. Il ressort que la pratique du mariage précoce surtout en milieu rural justifie cette sexualité précoce malgré le recul de l’âge au mariage. En effet, $45 \%$ des filles et $34 \%$ des garçons de 15-19 ans ont eu des rapports sexuels.

Chez les filles, les relations sexuelles surviennent généralement dans le cadre du mariage, alors que chez les garçons, elles surviennent en dehors du mariage. Selon The Alan Guttmacher Institute $^{7}$, à l'âge de 20 ans, 92\% des jeunes femmes et 52\% des jeunes hommes ont eu des relations sexuelles ; l'âge médian lors du premier rapport sexuel est de 17,2 ans pour les femmes et de 19,7 ans pour les hommes.

Il ressort également qu'essentiellement deux formes de sexualité précoce sont mises en exergue pendant l'adolescence. Il s'agit d'une sexualité très précoce légitimée socialement par le mariage et une sexualité encore proscrite en dehors du mariage.

A l'instar de ces constats, les résultats de cette enquête qualitative font ressortir effectivement la prédominance d'une sexualité précoce chez les adolescentes mariées ou non dans les cinq régions visitées. Les données montrent que la moyenne d’âge au premier rapport sexuel pour les adolescentes enquêtées se situe à 15,9 ans.

Si cet âge moyen est comparé au premier rapport sexuel avec l'âge moyen au mariage qui se situe à 15,5 ans, l'hypothèse selon laquelle les mariages précoces entraînent les rapports précoces est largement vérifiée.

Cette sexualité est vécue de manière " désordonnée » du fait de la naïveté, de l'ignorance, de la pauvreté, du «vouloir faire comme les autres" mais, surtout des mutations sociales et globalisantes qui ont abouti à un relâchement des mœurs.

\footnotetext{
${ }^{7}$ The Alan Guttmacher Institute : Les Adolescents au Burkina Faso : Santé Sexuelle et Reproductive Recherche en Bref 2004 Série, N³ 12/2004
} 


\subsubsection{Aperçu de la sexualité des adolescentes dans les différentes régions}

La sexualité des adolescentes se caractérise au Sahel par une extrême précocité et cela prend de l'ampleur malgré son caractère tabou. Comme l'affirme la sage-femme du CSPS urbain de Dori en ces termes : "Au Sahel, on dit que le sexe est tabou, mais c'est leur quotidien ». En général, cette précocité a pour conséquences des grossesses précoces et rapprochées.

Au Centre-Nord, les services de santé de Kaya font ressortir une recrudescence des grossesses des adolescentes de moins de 17 ans liée surtout au manque d'information. A Noaka, la sexualité précoce concernerait même les adolescentes de 12 ans selon l'accoucheuse auxiliaire. Ce qui entraîne des accouchements difficiles au regard de l’âge très précoce.

Dans le Centre-Est, les services de l'Action Sociale déplorent et mettent en exergue une sexualité des adolescentes affichée au grand jour, une sexualité mal gérée et surtout banalisée dans la localité. Les lieux publics tels que les "maquis ", les buvettes et les bars demeurent les endroits propices où se pratique une sexualité sans tabou ni gêne :

"On voit les adolescentes qui s'affichent au grand jour dans les bars, les buvettes et les chambres de passe...Il y a des grands hôtels ici qui attirent l'organisation de manifestations diverses avec des conséquences sur la sexualité des adolescentes. Ces conséquences se répercutent dans nos services à travers l'enregistrement des grossesses non désirées, des problèmes de paternité dont $20 \%$ sont référés à la justice...Des filles sont chassées de chez elles suite à ces types de grossesses, etc., (Action Sociale, Tenkodogo).

A Ouargaye, les agents de Santé et de l'Action Sociale soulignent que les adolescentes ont des informations sur la sexualité, mais elles ne les utilisent pas. Ce qui a pour conséquences les grossesses non désirées, des accouchements difficiles surtout chez les adolescentes non mariées. Les IST, comme l’a révélé l’ICP du CSPS urbain de Ouargaye, sont courantes:

"Nous recevons beaucoup de demande d'avortement...nous rencontrons aussi les cas d'IST comme la chaude pisse aussi bien chez les filles que les garçons », (ICP, CSPS Urbain de Ouargaye).

A l’Est également, cette sexualité des adolescentes, très développée, a été évoquée par les services de l'Action Sociale. Elle s'apparente à de la "prostitution occasionnelle » selon eux. Cette sexualité est également renforcée par des pratiques néfastes comme l'enlèvement des filles, le mariage précoce et forcé.

A l'instar des autres régions, celle du Centre-Sud présente les mêmes caractéristiques au niveau de la sexualité des adolescentes. Selon les agents de santé, leur sexualité est dynamique et précoce avec des comportements à risques, ce qui les expose à des maladies et infections opportunistes :

« Des cas d'IST sont signalés sur des sujets âgés de 15 à 16 ans. Il s’agit de la Gonococcie la syphilis, les candida albicans, les trichomonas vaginalis, etc. Les élèves seraient les plus touchés par les IST, les adolescentes non scolarisées par les grossesses non désirées et des demandes et tentatives d'avortement », (Maïeuticien d'Etat, CMA, Manga). 
Les données collectées à l’Action Sociale abondent aussi dans ce sens :

"Il y a un certain laisser-aller en matière de sexualité surtout chez les vendeuses ambulantes. La sexualité est banalisée jusqu'au niveau familiale et coutumier. Une femme mariée qui revient dans sa famille d'origine est libre de faire ce qu'elle veut de sa sexualité. Elle peut ainsi flirter avec qui elle veut. » (Action Sociale, Manga).

La sexualité précoce est généralement suivie d’une grossesse précoce de l’adolescente, le plus souvent immature physiquement, dont la structure osseuse, le bassin et les organes génitaux continuent à se développer. Ces grossesses précoces sont récurrentes dans la zone d’intervention du projet.

\subsubsection{Connaissances sur la sexualité et besoins en informations}

Il existe un déficit en matière de conseils et d'informations sur la gestion de la sexualité, de la puberté, des menstrues et de la contraception. La famille, la communauté, l'école, les institutions religieuses ne préparent pas suffisamment les adolescentes à une sexualité responsable. D’une façon générale, les adolescentes se forment et s’informent sur le tas à travers leurs expériences personnelles.

La faiblesse d'une éducation sexuelle se justifie par une absence d'échange direct, ouvert, explicite et spontané entre les parents et leur progéniture. En d'autres termes, la plupart des difficultés d'échange viennent du fait que la sexualité, dans le système traditionnel, est toujours tabou, surtout lorsque les adultes sont face aux enfants. Le mutisme sur la sexualité demeure très fortement ancré dans les us et coutumes aussi bien en ville qu'en campagne. Par conséquent, informer ou enseigner aux adolescents la sexualité reste problématique pour les parents qui ne se sont pas suffisamment outillés pour le faire.

Les résultats de l'étude ont également mis en exergue la faiblesse de communication des parents avec leurs enfants sur la santé sexuelle et reproductive. Les adolescentes mariées ou non et non scolarisées des cinq régions affirment ne pas aborder la question de la sexualité avec leurs parents qui ne veulent pas en parler et du coup ne leur en donnent pas l'occasion. Ils préfèrent laisser les adolescentes le découvrir par eux-mêmes.

Par contre, une minorité de célibataires et de scolarisées affirme aborder certains volets de la sexualité avec leurs parents, surtout les mères. Il s'agit essentiellement des questions sur les menstrues. Il ressort donc que le sujet de la sexualité n’est pas du tout discuté au niveau de la famille.

De même que les parents, les personnes âgées de la communauté n’échangent pas également sur la sexualité avec les adolescentes pour les aider à prévenir les grossesses non désirées et les IST. Pour les anciens, la communication autour de la sexualité est perçue comme un encouragement vers une sexualité socialement proscrite. Les personnes âgées sont prêtes à brimer facilement ceux ou celles qui la pratiquent; leurs faits et gestes sont soumis à un contrôle social très rapproché de la part des parents, des proches et même de toute la communauté comme le soulignent ces propos : 
«Cela est mal vu si on veut poser des questions sur la sexualité. Les parents disent que cela nous dépasse... ", "Si je pose de telles questions (à ma mère), elle va dire que je suis impolie » (Focus adolescentes, Dori)

"Un jour, j'avais mal au bas-ventre et je me suis rendue à la maternité où j'ai rencontré ma tante. Elle a tout de suite filé à la maison chez nous pour rapporter à ma mère. Heureusement que c'est ma mère qui m'avait dit d'aller au dispensaire pour faire voir mon bas-ventre. » (Focus adolescentes, Dori).

Par ailleurs, les services de Santé et de l'Action Sociale auxquels les adolescentes pouvaient s'adresser pour obtenir des informations sur la santé sexuelle et reproductive ne sont pas suffisamment outillés pour le faire. De plus, ces adolescentes ne savent pas que ces structures sont à même de leur fournir des informations sur la sexualité. Celles qui le savent n'y vont pas par pudeur ou de peur de rencontrer des parents et connaissances.

"Les adolescentes n'ont pas d'informations sur les services disponibles au CSPS en dehors des soins sanitaires. Les filles ne viennent ici qu'en cas de grossesse. Les garçons ne viennent même pas... » (Sage-femme, CSPS urbain Dori)

"C'est rare que les adolescentes viennent chercher des informations au CSPS car elles se gênent. » (Sage-femme, CSPS urbain II Tenkodogo)

L’enquête réalisée dans les cinq régions indique que la plupart des adolescentes mariées disent n'avoir pas eu d'informations sur la sexualité avant leur mariage. Elles reconnaissent aussi avoir développé très peu d'initiatives pour en chercher, du fait du caractère tabou du sujet, de la honte d'en parler, mais aussi de la faiblesse des structures susceptibles de leur donner ces informations. En effet, compte tenu de leur âge, les adolescentes soulignent qu'il leur est interdit d'écouter des informations sur la sexualité. Elles développent ainsi un complexe de soumission et de dépendance associé à la honte, qui freine en elles toute initiative de recherche d'informations. Certaines adolescentes par contre justifient leur ignorance des informations sur la sexualité par le fait qu'elles ne savent où aller les chercher.

La conjugaison de tous ces éléments détermine le fait que les adolescentes arrivent au mariage sans avoir suffisamment d'informations sur la sexualité.

«...je ne savais rien sur la sexualité...je n'ai pas cherché à savoir aussi parce que c'est comme cela ici...on ne pose pas de questions" (Adolescente mariée en grossesse, 16 ans, Mossi, musulmane, Dori).

«Je n'avais aucune information sur la sexualité... parce que j'avais honte de demander et de poser des questions sur un tel sujet», (Adolescente mariée post-partum, 17 ans, Mossi, Musulmane, Tenkodogo).

Les adolescentes non mariées sont davantage concernées par ce manque d'informations sur la sexualité tandis que les adolescentes dans les lycées et collèges expriment un assez bon niveau de connaissance sur la sexualité. Parmi leurs sources d'informations figurent les cours de Science de 
la Vie et de la Terre (SVT) et de puériculture, la radio, les séances de sensibilisation réalisées par l'Action Sociale, les services de santé, les associations et autres projets et programmes sur la SR. Les informations détenues par les adolescentes portent sur les thèmes liés à la connaissance des appareils génitaux, aux IST/Sida, aux menstrues, aux grossesses non désirées, au mariage précoce, etc.

L'étude montre que les conjoints des adolescentes, surtout ceux qui sont scolarisés, ne se gênent pas à aborder le sujet de la sexualité avec leurs conjointes. Ils affirment l'aborder à travers la planification familiale, l'éducation des enfants, les IST, les rapports sexuels, etc.

«On parlait de sexualité. On se disait qu'on allait faire la PF après l'accouchement. On a fait la piqure de trois mois. » (Mari adolescente post-partum, 19 ans, Mossi, protestant/Manga).

"On parle de sevrage de l'enfant, de rapports sexuels pendant la grossesse, du changement de position pendant l'acte sexuel.» (Mari adolescente en grossesse, 24 ans, Gourmantché, protestant/Bogandé).

"Oui. On échange sur les IST. On se raconte nos vies intimes. On parle de PF en disant de limiter les naissances à cause de la dureté de la vie. » (Mari adolescente post-partum, 32 ans, mossi, musulman/Bissiga).

Cependant, la majorité des adolescentes contrairement aux maris reconnaît n’avoir pas échangé sur le sujet avec leurs conjoints du fait de la honte et aussi de l'absence d'initiative à engager la conversation. Cela dénote de la gêne que les adolescentes éprouvent à évoquer le sujet plus que les hommes.

"Il n’y a pas d'échanges sur la sexualité avec mon mari. Il ne me demande pas, moi aussi je n'aborde pas le sujet à cause de la honte. " (Adolescente en grossesse, 17 ans, Gourounsi, musulmane/Tibilindi)

Quant aux besoins d'informations sur la sexualité ils sont déterminés par l'environnement socioculturel dans lequel sont conditionnées les adolescentes. Cet environnement socioculturel fait que la majorité des adolescentes surtout mariées trouvent que c'est normal de ne pas avoir ces types d'informations avant le mariage. Elles ne développent pas non plus des initiatives pour en chercher. Dans le jeu social, elles apparaissent comme des sujets non agissant, soumis d'une part aux décisions de leurs aînées, mais aussi à celles de leur mari.

Il existe également un réel problème d'expression et de formulation des besoins en informations sur la sexualité chez les adolescentes ${ }^{8}$, comme le dit celle-ci : « (...) je veux savoir quelque chose, mais je ne sais pas quoi » (Adolescente en grossesse, 16 ans, Mossi, Musulmane, Dori) ou encore "C'est à vous de savoir ce qu'il faut nous dire et qui peut nous aider (...) " (Adolescente en grossesse, 17 ans, Mossi, Musulmane, Noaka).

\footnotetext{
${ }^{8}$ Il y a beaucoup de non spécifications dans les réponses
} 
Les adolescentes enquêtées ont exprimé un ensemble de besoins qui touchent plusieurs aspects de la SR, ce sont des besoins en:

- informations et conseils sur la sexualité et la gestion de la sexualité ;

- informations sur la grossesse et la sexualité pendant la grossesse ;

- informations sur l'accouchement, l'hygiène et les soins au nouveau-né, et la sexualité après l'accouchement ;

- informations sur la planification familiale.

Pour les conjoints des adolescentes surtout les plus jeunes, des besoins d'informations en SR notamment sur la grossesse la sexualité du couple pendant la grossesse, la PF ont été formulés.

\subsubsection{Vécu de la sexualité}

Toutes les adolescentes mariées disent avoir eu leur premier rapport sexuel dans le mariage. Par contre, celles non mariées évoquent des occasions et des lieux divers. Il ressort des entretiens et des focus que la majorité des premiers rapports surviennent de façon inattendue chez ces dernières qui ne sont ni psychologiquement, ni physiquement préparées à l'acte. Ces rapports ont eu lieu lors des occasions festives (mariage, fête de fin d'année, Tabaski, Ramadan) comme le soulignent ces propos :

"C'est lors d'un pique-nique que j'ai eu mes premiers rapports sexuels. Je l'ai fait parce que je pensais que j'étais prête sans avoir pensé aux conséquences. » (Focus adolescente post-partum, Dori).

" J'ai eu mon premier rapport sexuel à 15 ans lors d'une sortie de 31 décembre. A cet âge, il ne m'était pas permis d'amener un garçon à la maison, donc je sautais le mur pour aller le rejoindre. » (Focus adolescente post-partum, Bogandé).

"Il m'a invité à sortir, nous sommes allés dans une buvette (appelée Chez Jackie) et après nous sommes allés chez lui et il y a eu le rapport sexuel », (Adolescente en grossesse, 19 ans, Gourmantché, Protestante, Manni).

De nos jours, les adolescentes vivent dans un contexte marqué par l'effritement des valeurs traditionnelles. L'exposition à d'autres cultures, l'acquisition d'une certaine indépendance ont eu entre autres pour conséquences une augmentation de l'activité sexuelle dans un contexte de restriction sociale, d'absence de dialogue, de conflits intergénérationnels et de pauvreté. Outre le fait que l'entrée en activité sexuelle est précoce et intense, elle est débridée et jugée à risque chez un grand nombre d'adolescentes car souvent associée aux relations non protégées.

Chez les adolescentes mariées, la pudeur entoure toujours cette question et elles ont des difficultés à parler de leur vécu de la sexualité. La majorité d’entre elles affirme ne pas avoir de problèmes dans le vécu de leur sexualité au sein du couple. Les quelques rares problèmes évoqués par certaines concernent la fréquence des rapports : 
"Mon mari a des rapports sexuels avec moi tous les jours, je lui ai dit de diminuer et il continue bien que je sois enceinte. » (Adolescente en grossesse, 15 ans, Mossi, Protestante/Bissiga).

Quant aux adolescentes célibataires, quelque soit leur appartenance ethnique, religieuse ou géographique, qu'elles soient instruites ou non, leur sexualité est vécue de façon désordonnée. En effet, la majorité d'entre elles dans leur quête de plaisir ont des rapports sexuels occasionnels et non protégés. De ce fait, elles prennent d'énormes risques ayant pour corollaires, les grossesses et les infections sexuellement transmissibles.

Toutes les adolescentes enquêtées n'ont pas voulu parler des problèmes liés au vécu de leur sexualité. Ce sont les agents de la santé et de l'Action Sociale qui les ont mis en exergue. Ce sont: les grossesses non désirées et/ou précoces, le problème de paternité, les IST, les avortements clandestins. Ils justifient la présence de ces problèmes par le fait que les adolescentes possèdent peu de connaissances sur la sexualité et d'habileté à concevoir le futur et à anticiper les conséquences de leurs actes. De plus, le déficit en matière d'informations et de conseils sur la gestion de leur sexualité a une grande répercussion sur le vécu de leur sexualité.

\section{- Le désir d'enfant/la nécessité de procréation}

Le désir d'enfant n'est pas seulement personnel, il exprime aussi un besoin de conformité avec le milieu social. La grossesse de l'adolescente intervient dans les mois qui suivent son mariage. Satisfaire les parents (famille d'origine et belle-famille) en enfantant très rapidement est le vœu de la grande majorité des adolescentes mariées. Si la grossesse tarde à venir au-delà de 2 ans, l'entourage commence à se poser des questions sur la capacité de procréation de la nouvelle mariée ou à développer d'autres types de considérations.

«...ma famille sait que quand une femme se marie, c'est pour faire des enfants, on ne peut pas attendre, sinon les gens vont te critiquer » (Adolescente en grossesse, 19 ans, Mossi, Catholique, Tenkodogo).

"Quand j'ai su que j'étais enceinte, j'étais rassurée à l'idée que je pouvais avoir un enfant...tout le monde s'attendait à cela parce que je suis chez un mari » (Adolescente en grossesse, 15 ans, Mossi, Protestante, Bissiga).

"Si tu dures avant d'avoir un enfant, les gens vont te critiquer en disant que tu fais exprès en prenant des contraceptifs » (Adolescente en grossesse, 17 ans, Mossi, Musulmane, Noaka).

Selon les adolescentes, il n'existe pas de consensus pour la conception et les normes sociales exigent de concevoir aussitôt après être marié. La contraception n'est donc pas une chose envisagée avant la première grossesse. L'adolescente ne décide pas de sa fécondité tout au moins de sa première grossesse. Le désir d'enfant n'est pas discuté au sein du couple. Les maris en majorité affirment être les décideurs du choix du moment d'avoir un enfant car ce sont eux qui épousent les femmes.

Les entretiens réalisés avec les maris confirment cette tendance comme l’illustrent ces propos : 
«...on ne décide pas du moment, si elle est là c'est que c'est pour faire des enfants... », (Mari d'adolescentes post-partum, 50 ans, Mossi, Catholique, Bissiga).

Chez les adolescentes célibataires interviewées, l'enfant n'est pas forcément désiré. La naissance d'un enfant est pour la plupart d'entre elles, le produit d'un « accident ». Il convient de souligner que ces dernières utilisent très peu les contraceptifs même si elles en connaissent l'existence.

Même si les résultats de l'enquête ont montré que le désir d'enfanter à un moment donné n'est pas planifié par les adolescentes, le nombre d'enfants qu'elles souhaiteraient avoir est cependant bien déterminé.

\section{- Sexe et nombre souhaités d'enfants}

Le nombre d'enfants souhaité est important aussi bien chez les adolescentes que chez les maris. Ce nombre varie de 2 à 10 enfants avec une moyenne de 4 enfants par adolescente. Cette moyenne est valable aussi bien pour les adolescentes post-partum que celles en grossesse sans considération du lieu de résidence, de la religion ou de l'ethnie. Certaines adolescentes n'ont pas pu déterminer le nombre d'enfants souhaité. Elles disent «s'en remettent à Dieu pour la gestion de leur vie génésique » comme l’illustrent ces propos :

" Je ne sais pas, c'est Dieu qui déterminera le nombre d'enfants à avoir » (Adolescente en grossesse, 14 ans, Peulh, musulmane, Dori/).

« Tout ce que Dieu donnera sera bon pour moi». (Adolescente post-partum, 16 ans, Musulmane, Peulh, Saouga/).

Pour les hommes, le nombre d'enfants souhaité varie entre 2 et 6 enfants avec une moyenne de 4 enfants par mari. Certains par contre n'accordent pas d'importance au nombre d'enfants car pour eux, c’est Dieu qui décide.

On remarque que le nombre de garçons souhaité par les adolescentes et leur conjoint excède toujours celui des filles dans les cinq régions. Mais, dans aucun cas, le souhait exprimé n’exclu les filles. Certaines adolescentes fondent cette préférence masculine au regard de la situation défavorisée de la fille et de la femme dans leurs milieux mais aussi parce qu'elles les aideraient dans les travaux domestiques.

Cette préférence s'explique aussi par le fait que les garçons perpétuent la famille et prennent en charge les parents à la vieillesse, alors que la fille va se marier dans une autre famille.

" Je veux trois garçons car après le départ des filles, ce sont eux qui restent pour aider les parents dans les travaux champêtres ou vont en aventure pour ramener de quoi prendre en charge toute la famille » (Adolescente en grossesse, 17 ans, Gourounsi, Musulmane, Tibilindi).

" Je désire 4 garçons (sur 5 enfants) car ils assurent beaucoup de travaux comme cultiver, garder les bœufs, traire les animaux et se marier à d'autres filles qui vont venir m'aider dans mes travaux domestiques » (Adolescente post-partum, 17 ans, Peulh, musulmane, Tibilindi). 
La faiblesse du nombre de filles souhaité répond aussi à d'autres raisons liées au contexte socioéconomique difficile surtout pour les couches vulnérables dont font partie les femmes. C'est ce qui est ressorti de certains entretiens individuels ou les adolescentes ont évoqué les difficultés pour l'éducation des filles et les différents problèmes de SR auxquels elles sont confrontées.

« Je veux seulement deux filles parce qu'il est difficile de nos jours d'éduquer une fille...de lui trouver un mari... », (Adolescente en grossesse, 17 ans, Mossi, Musulmane, Kaya).

«...les filles peuvent avoir une grossesse non désirée et voir ainsi leur avenir compromis...», (Adolescente post-partum, 17 ans, Mossi, Catholique, Tenkodogo).

Le nombre moyen d'enfants souhaité (4) par les adolescentes et leurs conjoints est certainement la résultante des différentes campagnes de sensibilisations sur la PF réalisée par les acteurs intervenant dans le domaine de la SR.

\section{- Sexualité des adolescentes pendant la grossesse}

La majorité des adolescentes disent ne plus avoir de rapports sexuels durant leur grossesse mais ne considèrent pas ce fait comme une interdiction et trouvent cela normal. Les adolescentes et les maris reconnaissent qu'il n’y’a pas d'échange entres eux sur la question avant d'arrêter les rapports sexuels pendant la grossesse.

Les adolescentes en grossesse et post-partum n’ont pas pu déterminer avec exactitude le moment de l'arrêt des rapports sexuels dû au fait que certaines adolescentes ne savaient pas l'âge de leur grossesse ou avaient honte d'aborder ce sujet.

Quant aux époux, certains n’ont pas souhaités abordés cette question avec l'équipe qui à leur avis est personnelle. D'autres arrêtent les rapports sexuels par crainte de la fausse couche. Cette croyance est liée à des considérations qui laissent entendre que le sperme peut entraîner de fausses couches.

"J'ai arrêté d'avoir des rapports sexuels avec ma femme dès qu'elle est tombée enceinte car je ne sais pas ce que cela peut faire au bébé... » (Mari d'adolescente post-partum, 24 ans, Malébé, musulman/Saouga).

«Quand j’ai su que ma femme était enceinte, j’ai tout arrêté... » (Mari adolescente post-partum, 22 ans, Mossi, catholique/Bissiga).

Il ressort des entretiens qu'il existe au niveau des adolescentes et de leurs maris une grande préoccupation par rapport à la sexualité du couple pendant le temps de la grossesse. Cette préoccupation s'est traduite par l'expression de besoins en information de la part des conjoints sur le sujet.

Leur souhait est de savoir comment avoir des relations sexuelles avec une femme lorsqu'elle est enceinte sans la faire souffrir. Quel est le moment idéal pour l'arrêt des rapports sexuels pendant la grossesse. Le besoin de s'informer sur les effets des rapports sexuels sur l'évolution du fœtus, sur les risques que la mère et le bébé encourent est une réalité chez les conjoints. 
Cependant, une minorité de maris affirme continuer les rapports sexuels jusqu'au terme de la grossesse.

«J'ai continué les rapports sexuels avec ma femme jusqu'à un mois avant l'accouchement », (Mari d'adolescente post-partum, 50 ans, catholique, Mossi, Bissiga/Boulgou).

\subsection{Grossesse chez les adolescentes}

Au Burkina Faso, plus de 25\% des jeunes femmes âgées de 15 à 19 ans ont déjà eu au moins une grossesse et $20 \%$ ont eu un enfant. ${ }^{9}$ A l'âge de 17 ans, une jeune femme sur cinq a eu un enfant ou est enceinte de son premier enfant. A l'âge de 20 ans, ce chiffre monte à près de trois sur cinq. L'ampleur du phénomène est beaucoup plus perceptible en zone rurale que dans les villes. En effet, le pourcentage d'adolescentes qui ont eu un enfant en zone rurale est deux fois plus élevé qu'en zone urbaine, soit respectivement $23 \%$ et $11 \%$. Ce qui dénote de l'ampleur du mariage et des grossesses précoces en milieu rural plus qu’en zone urbaine.

Ces chiffres montrent que la fréquence des grossesses chez les adolescentes est un phénomène récurent dans la plupart des communautés au Burkina Faso. Les perceptions et l'imaginaire populaire qui entourent la grossesse sont surtout basés sur le statut matrimonial de la femme plutôt que son âge. Ces perceptions influencent le vécu et la gestion de la grossesse chez la femme en général et chez l'adolescente en particulier.

D’une manière générale, le caractère valorisant de la grossesse des adolescentes prend le pas sur les risques immédiats et à long terme au regard de leur jeune âge. Si ailleurs, la grossesse des adolescentes est considérée comme un problème de santé publique et traitée comme tel, ce n'est pas encore le cas au Burkina Faso. Au contraire, elle fait partie des normes sociales de presque toutes les communautés quand elle intervient dans le cadre du mariage. Cependant, l'Etat et les partenaires au développement entreprennent des actions à l'endroit de la problématique.

\subsubsection{Découverte de la grossesse}

Les signes de la grossesse suscités constituent pour les adolescentes les éléments essentiels qui indiquent qu'elles sont enceintes. Cependant, certaines d'entre elles ont été informées de leur grossesse par leurs belles-mères, leurs mères ou autres personnes âgées de leur entourage. Quelques unes ont appris leur état de grossesse au cours d'une consultation médicale au CSPS pour d'autres problèmes.

"C'est à la suite d'une consultation pour cause de maladie que les agents de santé m'ont dit que je suis en grossesse » (Adolescente en grossesse, 17 ans, Mossi, Catholique, Noaka).

Selon les adolescentes elles mêmes et les agents de santé, le recours pour les tests de grossesse n'est pas une pratique courante dans les zones de l'étude.

La découverte de leur grossesse occasionne un sentiment de joie chez la majorité des adolescentes mariées dans toutes les communautés des cinq régions. Cette joie vient du fait

\footnotetext{
${ }^{9}$ Source Guiella G., santé sexuelle et de la reproduction des jeunes au BF : un état des lieux, 2004, Nº.12
} 
qu'elles sont devenues «femme », qu'elles sont capables de procréer et qu'elles ont la preuve de leur féminité comme le confirment ces propos :

« Je suis très contente et rassurée à l'idée que je pouvais tomber enceinte », (Adolescente en grossesse, 15 ans, Mossi, Protestante, Bissiga).

" J'étais contente parce que j'allais avoir un enfant comme les autres femmes », (Adolescente en grossesse, 19 ans, Mossi, Musulmane, Bissiga).

La survenue d'une grossesse est une étape capitale dans la vie d'une femme. En effet, selon ROVOLODOMANGA Bodo, " la grossesse est un événement capital dans la vie d'une femme » et LALLEMAND Suzanne la qualifie de «moment d'espoir » à cause de son caractère valorisant. La grossesse est une phase transitoire vers l'accomplissement et l'acceptation totale du statut de la femme dans la plupart des communautés. Le fait de tomber enceinte les rassure.

Cependant, il ressort des réponses des adolescentes enquêtées que cette joie est quelquefois mélangée à une sorte de crainte pour l'issue de la grossesse, de souci de sa gestion. Les propos de cette adolescente illustrent cette réserve :

"...Je suis contente certes, mais de façon mesurée parce que je n'ai pas encore accouché, on ne sait jamais », (Adolescente en grossesse, 19 ans, Mossi, Catholique, Tenkodogo).

Chez les adolescentes non mariées, cette crainte est aggravée par la difficulté de gestion de la grossesse et par la stigmatisation dont elles sont victimes. La découverte de la grossesse constitue une source d'angoisse supplémentaire pour elles à cause souvent de sa non acceptation par les parents. Le récit de vie suivant montre la situation difficile que vit une adolescente rejetée par sa famille et l'auteur de sa grossesse.

« Je ne disposais pas d'information sur la sexualité. J'entendais parler du sujet et les gens disaient que si tu couches avec un homme tu vas avoir une grossesse. J'ai rencontré un jeune paysan de 20 ans avec qui j'ai eu des relations sexuelles. Au début il utilisait la capote et ensuite il m'a dit qu'il ne peut plus continuer parce que ce n'était pas bon et que, si je tombe enceinte, il me fera avorter. Quand je suis tombée enceinte, il n'a pas tenu parole. Mes parents veulent me faire avorter mais le marabout leur a dit que si j'avortais, j'allais mourir. Néanmoins, ils m'ont obligé à mettre des médicaments dans le sexe, cela m'a fait des plaies partout mais je ne suis pas allée au dispensaire. Je lavais les plaies au savon et je mettais du beurre de karité. Ils veulent toujours me faire avorter mais je refuse. Si ça continue, je vais prendre des médicaments pour mettre un terme à tout cela (me suicider). Je regrette maintenant car mon copain ne veut pas m'accueillir chez lui et me parle mal. J'ai trop de problèmes avec mes parents qui sont devenus méchants avec moi, ils me traitent comme une chienne et ne me donne plus rien, je vais laisser tomber mes études et attendre l'année prochaine peut-être. » (Adolescente, 19 ans, 4 mois de grossesse, élève, Manni)

Une fois découverte, la grossesse n'est pas rendue publique de manière systématique du fait des normes sociales, de la honte et de la gêne qui ne permettent pas aux adolescentes de faire de telles annonces. Mais, dans la plupart des communautés enquêtées, les belles-mères sont les premières informées et jouent un rôle important dans la révélation de la découverte de la grossesse. Elles 
annoncent l'existence de la grossesse d'abord à leurs fils (conjoints des adolescentes), ensuite dans le cercle familial restreint puis au delà.

Il existe également dans les communautés d'autres comportements évocateurs d'une grossesse au niveau familial. Chez certains musulmans par exemple, quant la femme prie de manière ininterrompue, la famille sait qu'elle ne voit pas ses menstrues et déduit donc qu'elle est enceinte. Chez les Peulhs, en plus de ce fait, elle ne s'occupe plus du pâturage durant toute la grossesse, car c’est une activité contraignante.

Outre ces comportements évocateurs, dans d'autres groupes mossi par exemple, on relève certains rituels qui servent non seulement à annoncer la grossesse au cercle familial, mais aussi aux autres membres de la communauté. Chez les Mossi de Bissiga par exemple, une cérémonie appelée couramment par la population en langue zoaga " wuusa puga» qui signifie « annonce de la grossesse " est organisée pour rendre officielle la grossesse au couple et à l'entourage. Elle consiste à verser faire verser de l'eau par surprise et par un enfant sur le couple, tard dans la nuit pendant son sommeil. Le lendemain, un mets spécial est préparé et partagé à l’intérieur de la concession et dans tout le quartier. C'est à l'issue seulement de cette cérémonie que la grossesse est rendue officielle, et que les dispositions qui doivent être prises pour son bon déroulement sont envisagées y compris le suivi médical au centre de santé. Ces propos d'une adolescente résument le déroulement de cette cérémonie.

"Ma grossesse a été annoncée à la belle famille à travers un rituel. Pendant que nous dormions encore mon mari et moi, nous avons été arrosés d'eau fraîche une nuit tard vers 4 h du matin par une femme choisie pour la circonstance...au matin venu, on a préparé du tôt à la sauce haricot qu'on a partagé à l'intérieur de la concession et dans tout le quartier. Comme cela tout le monde est informé ». (Adolescente en grossesse, 19 ans, Mossi, Musulmane, Bissiga).

Ces pratiques tendent à disparaître car de nos jours, dans la plupart des communautés, l'annonce de la grossesse se fait plus librement. Il convient de souligner que souvent, ces cérémonies peuvent se dérouler plusieurs mois après le début de la grossesse, et par conséquent, entraînent un retard dans son suivi médical. Une partie de l'entourage apprend la grossesse par elle-même à travers le développement du ventre de l'adolescente.

\subsubsection{Vécu de la grossesse}

A quelques exceptions près, la majorité des adolescentes sont tombées enceintes quelques temps seulement après leur mise en couple. Pour certaines d'entre elles, la grossesse est survenue rapidement quelque fois de manière inattendue. Le vécu de la grossesse chez l'adolescente est influencé par plusieurs facteurs d'ordre socio-culturel, économique mais aussi par le niveau de connaissances qu'elles ont sur le sujet.

Dès la découverte ou l'annonce de la grossesse, les adolescentes deviennent le centre de toutes les préoccupations dans leurs belles-familles et familles d'origine. Elles affirment dans leur majorité avoir observé des changements de comportements chez leurs proches qui se sont traduite par des soutiens et des gestes de solidarité divers. Comme témoignage, elles déclarent que leurs désirs alimentaires sont satisfaits. En plus de ce soutien, elles bénéficient d'un environnement propice fait d'ambiance et de bonne humeur nécessaire au bon déroulement de leurs grossesses. 
Les adolescentes non mariées dans la plupart des cas vivent différemment leur grossesse. Leur état est perçu dans les communautés comme un phénomène hors norme. Il s’agit, dans la plupart des cas, de grossesses non désirées où le problème de la paternité se pose. Cette situation s'accompagne souvent de tentatives d'avortements, d'abandons scolaires ou de formation professionnelle, entraînant la perte de confiance en elles-mêmes.

La revue littéraire a fait ressortir également que la grossesse est souvent représentée comme une charge et une source d'angoisse pour l'adolescente. Outre son caractère valorisant, LALLEMAND Suzanne notifie que la grossesse est paradoxalement un moment de péril et d'angoisse eu égard aux risques encourus par la mère et l'enfant. Il en ressort également que les adolescentes sont dans une période de transition de leur développement où la grossesse et le mariage constituent des sources de stress émotionnels supplémentaires.

Les belles-mères et les maris rencontrés disent se focaliser sur les soutiens à apporter aux adolescentes dès le début de leur grossesse. Ils affirment apporter le nécessaire alimentaire à l'adolescente, être à ses petits soins et éviter de la contrarier pour le bon déroulement de la grossesse.

\subsubsection{Rituels, restrictions et conseils liés à la grossesse}

La prise en charge de la grossesse, d'un point de vue traditionnel consiste à la pratique de rituels et coutumes pour mener à bien la grossesse et à l'observance rigoureuse des interdits, restrictions et conseils. Dans la plupart des cas, ce sont les parents qui informent les adolescentes de ces rituels et interdits dont la signification leur échappe généralement.

Les données de notre enquête révèlent l'existence de deux types de rituels dont un religieux et l'autre culturel. Les rituels d'ordre religieux à propos de la grossesse ont surtout été évoqués par les leaders religieux qui consistent à prier sur la grossesse afin de la bénir et de lui assurer un bon déroulement.

Les rituels relevant du domaine culturel se traduisent par des ports d'amulettes données par les belles-mères et autres vieilles personnes de l'entourage des adolescentes en grossesse qui ignorent souvent leur signification précise.

Mais, il faut souligner que la plupart des adolescentes interrogées affirment n'avoir pas subi de rituels ni à la découverte de leur grossesse, ni au suivi de celle-ci pour des raisons religieuses notamment. En effet, l'appartenance à l'Islam et au Christianisme expliquerait en partie la faible pratique de ces rites dans la plupart des communautés visitées. L'appartenance à ces religions serait incompatible avec la pratique des rites traditionnels comme l'affirme cette adolescente :

«Nous n'avons pas fait de rituels parce que nous sommes des chrétiens », (Adolescente en grossesse, 18 ans, Gourmantché, Protestante, Manni).

La revue littéraire fait ressortir d'autres rituels liés à l'ethnie qui visent à assurer un bon déroulement de la grossesse. L'étude sur l' "Itinéraire thérapeutique des adolescentes en grossesse et première fois parents», menée par Population Council en 2003, révèle des sacrifices 
et des vénérations des ancêtres communs aux Gourmantché et aux Mossis. Il ressort de la même étude que le recours aux devins et devins guérisseurs (tapeurs de sable, géomanciens chez les Gourmantché et marabouts chez les mossis).

La période de grossesse est reconnue également être une période de soumission à des interdits. Ceux-ci varient également selon les groupes ethniques et religieux. Plusieurs types d'interdits ont été évoqués dont les plus importants sont les suivants : les interdits d'ordre alimentaires et ceux liés à des restrictions comportementales et vestimentaires.

Les interdits alimentaires font ressortir qu'une femme en grossesse est tenue de suivre un régime alimentaire particulier pour, selon les personnes interrogées, assurer un accouchement facile et apporter à l'enfant, force, santé et intelligence. Ils concernent les aliments aigres, pimentés et amers «...qui ne plaisent pas à la grossesse » et les aliments de type sucré susceptibles de faire grossir le bébé et d'engendrer des difficultés au moment de l'accouchement.

Par ailleurs, des études sur la question soulignent aussi l'existence d'autres types d'interdits alimentaires auxquels sont soumises les adolescentes. C'est le cas où l'adolescente ne doit pas consommer de la viande de certains animaux pour éviter que l'enfant n'hérite des traits caractéristiques physiques ou comportementaux de ces animaux. Par exemple, la consommation de la viande de rat voleur rendrait l'enfant voleur, celle des pattes de crabes déformerait les jambes de l'enfant. La consommation de la viande de bœuf sans corne, rendrait plus tard l'enfant malchanceux et impuissant. Elle doit aussi éviter de consommer tout aliment associé au génie (poulet, miel, sucre, œuf) selon Doris BONNET (1988).

Pour ce qui est des restrictions comportementales et vestimentaires, les données recueillies soulignent également que l'adolescente évitera de sortir aux heures chaudes, de fréquenter les endroits habités par des génies. Les futurs parents ne doivent pas élever des pintades afin de mener à bien la grossesse. Il faut exécuter certaines tâches selon des moments bien précis et porter des habits qui cachent bien le ventre pour ne pas l'exposer aux regards d'autrui comme l'affirment ces propos.

"On m'a dit de ne pas exposer mon ventre nu aux regards des autres parce que des personnes peuvent vouloir me faire du mal », (Adolescente en grossesse, 17 ans, Peulh, musulmane, Tibilindi)

«Non, je n'ai pas subi de rituels, mais ne pas se doucher tard dans la nuit, ne pas sortir torse nu au dehors la nuit, ne pas porter des petits habits qui laissent le ventre dehors, ce sont les parents qui me l'ont dit », (Adolescente en grossesse, 14 ans, Bella, Musulmane, Dori).

L'analyse du régime alimentaire auquel les adolescentes sont soumises durant leur grossesse montre qu'il ne s'agit pas d'une alimentation basée sur les normes nutritionnelles recommandées par les agents de santé, mais sur l'expérience des belles-mères et les croyances communautaires en la matière. Aussi, cette restriction alimentaire peut ne pas être adaptée aux besoins réels des adolescentes pour un bon déroulement de la grossesse. 


\subsubsection{Charge de travail des adolescentes pendant la grossesse}

Le vécu de la grossesse s'accompagne également d'une modification de la charge de travail des adolescentes. Il s'agit plutôt d'un allègement des tâches dans les travaux champêtres et domestiques dans la majorité des sites de l’étude.

Certaines pratiques culturales telles que les labours exigent souvent des efforts physiques intenses. A cause de leur état fragile, les adolescentes en sont totalement ou partiellement déchargées suivant l'évolution de la grossesse. Elles continuent cependant d'assurer des tâches légères comme le semis et le désherbage.

Le deuxième niveau de l'allègement des tâches s'opère à travers les travaux domestiques. Ici également, les adolescentes sont exemptées des travaux durs consistant à piler, à puiser l'eau ou à chercher du bois comme en témoignent ces adolescentes :

" J'ai cessé de piler et de puiser l'eau à cause de mon état ». (Adolescente en grossesse, 17 ans, Peulh, musulmane, Tibilindi).

«...je ne vais plus au champ, je reste seulement à la maison ou je m'occupe des petits travaux domestiques» (Adolescente en grossesse, 19 ans, Mossi, musulmane, Bissiga).

Seule une minorité d’adolescentes déclare ne pas bénéficier de cet allègement de travaux durant cette période fragile de grossesse dans certains groupes ethniques. Il s'agit de certains groupes peulh (Bella, Malébé, Tamachèque notamment) où les femmes enceintes doivent travailler comme d'habitude, voire davantage pour faciliter plus tard leur accouchement.

"Ici, il faut travailler à mort jusqu'à l'accouchement ; il faut piler, puiser l'eau et chercher du bois...c'est comme ça ici, c'est pour faciliter l'accouchement». (Adolescente post-partum, 16 ans, Malébé, musulmane, Saouga).

L'allègement des tâches chez les adolescentes en grossesse met en exergue une certaine solidarité du groupe envers l'adolescente en grossesse. En effet, les belles-mères et d'autres femmes de la concession aident souvent l'adolescente dans les travaux champêtres et domestiques.

Quant aux maris, ils affirment tout mettre en œuvre pour assurer le bon déroulement de la grossesse de leurs femmes, en les déchargeant d'un certain nombre de travaux durs et en s'impliquant dans la réalisation des travaux domestiques.

«...Je la surveillais de près, je lui ai dit de ne pas faire les travaux lourds comme labourer, battre et piler le riz... » (Mari d'adolescente en grossesse, 32 ans, Mossi, musulman, Bissiga).

«Je l'aide à puiser l'eau du forage, je fais aussi la cuisine de temps en temps...» (Mari d’adolescente en grossesse, 24 ans, Gourmantché, protestant, Bogandé).

« Je lui ai dit de ne pas faire les travaux qu'elle ne peut pas faire et je l'aide à faire la lessive, la vaisselle et souvent la cuisine » (Belle-mère ado en grossesse, 52 ans, Gourmantché, catholique, Manni). 


\subsection{Itinéraires thérapeutiques des adolescentes pendant la grossesse}

\subsubsection{Itinéraires thérapeutiques}

Il s'agit dans cette partie de présenter une typologie des trajectoires suivies (itinéraires thérapeutiques) par les adolescentes depuis le diagnostic de la grossesse jusqu'à terme. Cette typologie permet d'analyser le suivi sanitaire des adolescentes et les conditions d'accès au centre de santé, en portant une attention particulière à la prise en charge de la grossesse sur le plan social.

D’une manière générale, les résultats de l'étude ont montré que les adolescentes mariées en grossesse ont les mêmes itinéraires thérapeutiques. En effet, la majorité des adolescentes ne vont pas systématiquement au centre de santé pour confirmer leurs grossesses, se basant sur les signes précurseurs et les constats de leurs belles-mères. Elles vont généralement tard au centre de santé pour la CPN, après le premier trimestre, accompagnées de leurs belles-mères pour la première fois.

Même si les adolescentes font un suivi régulier de la CPN, leur itinéraire est basé surtout sur les soins à domicile par l'automédication en cas de malaises ou de maladies quelconques. Elles se réfèrent à la médecine traditionnelle par l'utilisation de plantes et de décoctions reçues des bellesmères. Le centre de santé n'est que le dernier recours en cas de persistance de la maladie.

Quant à la minorité qui ne fait pas de CPN, elle a recours qu'aux tradipraticiens et aux marabouts en cas de maladies.

Ces itinéraires changent lorsqu'il s'agit des adolescentes non mariées. Une fois enceinte, la majorité d'entre elles tentent d'avorter le plus souvent à l'insu des auteurs ou en cas de refus de reconnaissance de la grossesse par les présumés auteurs. A cet effet, leur premier recours est aussi l'automédication par l'utilisation de plantes médicinales (feuilles de neem....), de produits nocifs (détergent, acide, potasse, bleue de lessive, etc.) ou de comprimés donnés par des connaissances. En cas d'échec, elles se rendent chez les tradipraticiens qui leur donnent des produits à boire, à purger et à mettre dans le vagin. Ce n'est qu'après plusieurs tentatives, qu'elles se rendent au centre de santé où elles reçoivent les conseils contraires à leurs projets. Ce qui est courant, c'est qu'elles ne reviennent plus au centre de santé, ou très peu y reviennent pour faire la CPN. Elles restent cachées avec leurs grossesses. Certaines d'entre elles arrivent à avorter, d'autres non. Parmi ces dernières, quelques unes accouchent au CSPS et les autres à domicile. A cause de la situation complexe dans laquelle les grossesses des adolescentes non mariées surviennent, il est difficile de tracer un itinéraire thérapeutique très précis de cette cible qui se cache du fait de la gêne qu'elles éprouvent vis-à-vis de leur état.

Ces itinéraires thérapeutiques sont déterminés par des croyances, des attitudes et comportements des adolescentes, des personnes ressources en charge de la grossesse. 


\subsubsection{Connaissances sur la grossesse et besoins en informations}

Il s’agit d'apprécier le niveau de connaissances des adolescentes, des belles-mères, des AV, des agents de santé et des maris. Pour chaque acteur, il s'agissait de faire ressortir le niveau d'informations sur les signes de reconnaissance et du bon déroulement d'une grossesse. Chez les adolescentes, d'autres éléments comme l'anatomie et l'entretien de la grossesse ont été pris en compte dans l'appréciation de leur niveau de connaissances.

Dans les cinq régions, il apparaît chez la majorité des adolescentes, une méconnaissance de la transformation de leur corps liée à l'avènement de la puberté. Certaines ne font pas la relation entre l'âge et l'exposition à des risques de grossesse en cas de rapport sexuel. Ainsi, plusieurs d'entre elles sont tombées enceintes parce qu'elles ignoraient leur maturité physique :

«...je ne savais pas qu'à 16 ans, on pouvait tomber en grossesse... » (Adolescente en grossesse, 17 ans, Gourounsi, Musulmane, Tibilindi).

La connaissance des signes évocateurs d'une grossesse et de son bon déroulement ou non permet aussi d'apprécier le type d'informations dont disposent les adolescentes.

Elles sont majoritairement, tout profil confondu, à citer :

- l'arrêt des règles

- La salivation abondante

- Le développement du ventre

- Les douleurs au bas-ventre

- Les nausées

- Le manque d’appétit

- L'éclaircissement du teint du visage

- La poussée des seins.

En revanche, elles sont majoritaires à reconnaître les signes du bon déroulement ou non de la grossesse, sans doute du fait de leur première expérience. Les conséquences qui découlent de cette situation sont entre autres, le suivi tardif et irrégulier de la grossesse et les tentatives d'avortements surtout en ce qui concerne les adolescentes non mariées.

Malgré ce faible niveau de connaissance, la recherche d'information n'est pas systématique par la majorité des adolescentes, même si elles sont conscientes de son importance dans la gestion de leurs grossesses. Les principales sources d'informations pour celles qui ont cherché à en connaître davantage sur la grossesse sont entre autres :

- les belles-mères en ce qui concerne les adolescentes mariées

- la mère, les tantes ou autres femmes de la famille, pour les adolescentes non mariées

- les amis (es)

- les médias telles que la radio, la télévision

- les cours dispensés au primaire (puériculture) et au secondaire (éducation à la vie familiale et Sciences de la Vie et de la Terre).

La majorité des adolescentes enquêtées ont affirmé avoir des besoins d'informations sur la grossesse même si elles restent imprécises sur ces besoins. Les réponses du type "je veux tout savoir », "c'est au projet de savoir ce qu'il faut faire » ou " je ne sais quoi demander » étaient récurrentes comme illustrent les propos suivants : 
"J'ai besoin d'informations sur la sexualité et la grossesse, mais je ne sais pas quoi précisément », (Adolescente en grossesse, 19 ans, Mossi, Catholique, Gogo).

«J'ai besoin d'informations sur tout ce que je ne connais pas sur la grossesse, ...tout ce que vous allez nous dire, il faut le dire pour qu'on apprenne nous aussi » (Adolescente en grossesse, 17 ans, Mossi, Musulmane, Gogo).

"Oui, je veux tout ce qui est bon pour ma santé, mais je ne peux pas choisir, c'est à vous de le savoir », (Adolescente en grossesse, 18 ans, Gourmantché, Protestante, Manni).

Pour celles qui ont pu s’exprimer avec plus de précisions, l'essentiel des besoins en informations se focalise sur les aspects suivants :

- l'entretien d'une grossesse

- la contraception

- l'alimentation pendant la grossesse

- les comportements et attitudes à adopter lors de la grossesse

- les soins pour assurer le bon développement du fœtus

- etc...

Plusieurs raisons expliquent cette insuffisance, voire ce manque d'information chez les adolescentes sur la grossesse, ce sont :

- L'absence de communication entre parents-enfants sur le sujet

- Le manque d'initiative des adolescentes pour rechercher l'information

- L'insuffisance des structures intervenant dans le domaine de la SR

- l'inadaptation des services de Santé et de l'Action Sociale aux besoins des adolescents.

Du coté des maris, ils évoquent comme signes indicateurs de grossesse les maladies fréquentes (fièvre, paludisme), le dégoût des aliments habituels, l'état d'abattement, comme l'affirment ces propos :

«J'ai commencé à soupçonner la grossesse parce qu'elle ne voulait plus des aliments habituels, elle sortait tardivement du lit...», (Mari d'adolescente post-partum, 50 ans, Mossi, Catholique, Bissiga).

Quelle que soit la localité visitée, il est apparu que les belles-mères ont une bonne connaissance des signes de manifestation d'une grossesse et de son déroulement ou non, du fait de leurs expériences en la matière. L’absence de douleur, de maladie, de saignement et d'écoulement au cours de la grossesse traduit selon elles son bon déroulement. La présence d'un de ces signes est un risque pour la grossesse.

Les AV ont également une bonne connaissance des signes de complications d'une grossesse au regard des formations reçues pour mener des sensibilisations sur les consultations prénatales et les signes de danger pendant la grossesse.

Mais, selon les agents de santé, d'autres signes sont également à prendre en compte pour l'identification d'une grossesse qui ne se déroule pas normalement. Il s'agit entre autres :

- des œdèmes aux membres inférieurs qui peuvent traduire un problème rénal

- de la fièvre

- des céphalées intenses qui peuvent révéler une baisse de tension artérielle 
- des vomissements gravidiques sévères qui peuvent être cause de déshydratation

- l'absence de mouvement actif du fœtus

- le retard de la croissance intra-utérine ou le non grossissement du fœtus

\subsubsection{Suivi sanitaire}

Quelle que soit la région visitée, on remarque que le suivi sanitaire des adolescentes en grossesse est basé surtout sur les soins à domicile. En fait, tout semble être une question de gravité du mal dont souffre l'adolescente. L'automédication par l'utilisation des plantes médicinales et/ou des médicaments prohibés (médicaments de la rue) est le premier recours de la majorité des adolescentes en cas de maladies et/ou de malaises. Le deuxième recours est le centre de santé en cas de persistance. Certaines associent les deux méthodes de traitement. Quelques-unes d'entre elles n’ont pas pu donner des informations sur un recours précis.

«Un jour où j'étais malade, j'ai acheté des comprimés au marché que j'ai avalés. Cela m'a soulagée... », (Adolescente en grossesse, 17 ans, Gourounsi, musulmane/Tibilindi).

«Quand je suis tombée malade, ma belle-mère a trempé une plante dans l'eau que j'ai bue... Après, je me suis sentie bien. » (Adolescente en grossesse, 15 ans, Malébé, musulmane/Saouga).

La majorité des adolescentes enquêtées affirment faire le suivi de leurs grossesses au centre de santé. Généralement, ce suivi se fait tardivement car il débute seulement après le premier trimestre (4-5 mois). La plupart des adolescentes en grossesse (surtout celles qui sont mariées) font la CPN régulièrement. Ce qui a été confirmé par les accoucheuses des cinq régions. Elles justifient ce suivi régulier par le fait que la population a été sensibilisée sur la fréquentation des centres de santé, mais aussi par la présence du SONU et la prise en charge complète par le projet HELP $^{10}$. Ces actions ont apporté une amélioration dans la fréquentation des centres de santé par les femmes en grossesse en général et les adolescentes en particulier. Il est donc important de souligner que la majorité des adolescentes mariées enquêtées dans les cinq régions fréquentent les centres de santé pour le suivi de la grossesse, contrairement à une minorité qui reconnaît n'avoir pas fait de CPN durant la période de leur grossesse par manque de moyens financiers ou par ignorance.

«Je ne suis pas encore allée au CSPS parce que l'auteur de ma grossesse ne m'a pas encore donné de l'argent » (Adolescente en grossesse de 6 mois, 17 ans, mossi, catholique, Manga).

«Je ne suis pas encore partie à l'hôpital pour le suivi car je n'ai pas d'argent... Je suis même malade depuis et on dit qu'il n'y a pas d'argent car mon mari n'a pas envoyé l'argent de la Côte D'Ivoire. Si jusqu'à l'accouchement, l'argent ne vient pas, je vais accoucher à la maison.» (Adolescente en grossesse, 14 ans, Bella, Musulmane/Dori).

\footnotetext{
${ }^{10}$ HELP : ONG française intervenant à Dori qui fait la prise en charge des soins obstétricaux et du nouveau-né jus qu'à 5 ans.
} 
«C'est à 7 mois que j'ai commencé la CPN car je ne savais pas qu'il fallait aller au centre de santé avant le jour de l'accouchement." (Adolescente post-partum, 17 ans, Mossi, Catholique/Kaya).

Le suivi sanitaire précédemment décrit change pour la majorité des adolescentes non mariées en grossesse qui n'ont recours au centre de santé que pour des complications d'avortement.

Il ressort de l'analyse des données collectées que les adolescentes qui ont fait la CPN s'orientent plus facilement vers un centre de santé pour les accouchements. Elles justifient ce recours par les conseils donnés par l'agent de santé lors du suivi de la grossesse.

" L'agent de santé m'a dit de venir à la maternité dès que j'ai mal au ventre » (adolescente en grossesse, 19 ans, Catholique/Manga).

Le suivi médical des adolescentes en grossesse au centre de santé a été facilité par l'appui de personnes clés telles le mari et/ou la belle-mère. En effet, pour la majorité des adolescentes n'exerçant pas d'activités professionnelles ou économiques et ne disposant pas de revenus réguliers, il est difficile d'assumer les frais du suivi médical. Aussi, l'apport de ces personnes a été déterminant dans l'évolution et l'issue de la grossesse.

La majorité des maris d'adolescentes enquêtés affirment avoir facilité le suivi médical de la grossesse de leurs épouses en mettant à leur disposition les moyens financiers pour honorer les frais relatifs à la CPN et aux ordonnances lors des maladies. Tous reconnaissent aussi le bienfondé du suivi médical de la grossesse et ont déclaré amener leurs épouses dans un centre de santé pour une meilleure prise en charge de la grossesse.

\subsubsection{Gestion de la grossesse des adolescentes et prise de décisions}

Plusieurs types d'acteurs sont impliqués dans la gestion de la grossesse des adolescentes. Ce sont :

- les acteurs au niveau familial qui se composent des belles-mères, des maris, des mères et des tantes

- les acteurs communautaires composés essentiellement des AV

- les agents de santé.

\subsubsection{Acteurs au niveau familial}

\section{Les belles-mères}

Il ressort des résultats de l'étude que les belles-mères sont les actrices principales de la gestion des grossesses chez les adolescentes mariées, la mère ou la tante pour celles non mariées. Ce sont des femmes âgées de plus de 50 ans, présentant une durée moyenne de plus de 35 ans d'expérience dans le mariage et la maternité. Ce statut leur confère naturellement le premier rôle dans la gestion des grossesses qui consiste généralement à encadrer l'adolescente du début de la grossesse jusqu'à la période post-partum. Ce rôle varie selon les différentes étapes d'évolution de la grossesse et peut se présenter ainsi qu'il suit :

- Au début de la grossesse : 
- $\quad$ aider l'adolescente à prendre conscience de son nouvel état

- aider l'adolescente à accepter sa grossesse

- donner les informations nécessaires à l'entretien de la grossesse.

- Au cours de la grossesse :

- donner les informations et conseils nécessaires au bon déroulement de la grossesse

- $\quad$ soutenir moralement et aider l'adolescente dans ces différents travaux

- Apprendre les bonnes attitudes et comportements à adopter

- Apprendre l'observation des mesures d'hygiène

- S'assurer de la réalisation des rituels

- Encourager et accompagner l'adolescente à faire suivre sa grossesse au centre de santé

- $\quad$ Assurer les frais liés au suivi et aux soins médicaux.

- Au terme de la grossesse :

- Préparer matériellement et financièrement l'accouchement

- Apprendre les bonnes attitudes et comportements à adopter lors de l'accouchement

- S'assurer de la réalisation des rituels.

Chez les Peulh, ces rôles sont partagés entre la belle-mère et la mère de l'adolescente mariée. L'adolescente reste dans la belle-famille du début de la grossesse jusqu'au septième mois, puis elle rejoint sa famille d'origine où elle y reste environ 3 à 4 mois après son accouchement.

\section{Les maris}

Le profil des maris d'adolescentes rencontrés dans les cinq régions montre qu'ils ont une moyenne d'âge de 25 ans dont le plus élevé à 50 ans. Ils sont dans l'ensemble monogames et sont dans leur majorité première fois parent. Ce qui dénote de leur manque d'expérience en matière de gestion de grossesse. Au - delà de cette réalité, les maris ne sont pas tellement impliqués dans la gestion des grossesses dans la plupart des communautés de la zone de l'étude. D'une manière générale, leurs rôles peuvent être résumés comme suit :

- soutenir moralement et aider l'adolescente dans ces différents travaux

- encourager et accompagner l'adolescente à faire suivre sa grossesse au centre de santé

- $\quad$ assurer les frais liés au suivi et aux soins médicaux

- préparer matériellement et financièrement l’accouchement.

D’autres acteurs comme les beaux- pères, les femmes des frères, les sœurs et autres femmes de la concession sont aussi impliqués dans la gestion de la grossesse de l'adolescente mais de façon moins prononcée. Si les beaux-pères assurent quelque fois les frais de suivi et de soins médicaux, les autres femmes aident surtout dans les travaux et soutiennent moralement l'adolescente. La gestion de la grossesse des adolescentes n'est pas du seul ressort de la cellule familiale. Il existe aussi des agents communautaires et des structures de santé. 


\subsubsection{Acteurs communautaires}

Il s'agit essentiellement des accoucheuses villageoises recrutées pour servir de relais entre les villages et les services de santé en matière de SR. Elles ont été installées par les autorités sanitaires qui les ont formées et équipées pour accoucher les femmes. Mais, au regard des limites de l'intervention de cette catégorie d'acteurs révélées par une mission d'évaluation du Ministère de la Santé en 2004 et de la nécessité que les accouchements soient effectués par un personnel qualifié de la santé, le rôle des AV a été réorienté pour se focaliser exclusivement sur les activités de sensibilisations et les tâches suivantes :

- sensibilisation/IEC

- préparation à l'accouchement

- soins au nouveau né à domicile

- distribution des produits et des services de base

- gestion/collecte des données

- notification de la naissance

- référence au centre de santé

- accompagnement des femmes au CSPS

- visites à domicile

- mobilisation sociale et organisation de la vaccination

- promotion de la nutrition

- prise en charge de certaines maladies (paludisme simple, diarrhée non compliquée).

Cette initiative a fait diminuer le nombre d'accouchements à domicile. Cependant, quelques AV continuent toujours d'accoucher dans les maternités pour palier à l'insuffisance de personnel comme c'est le cas à Gogo et à Noaka. Cette AV justifie le fait qu'elle continue toujours d'accoucher les femmes à travers ces propos :

"Je suis venue ici car il n'y avait que 2 agents de santé. On m'a dit de venir les aider », (AV, 50 ans, Mossi, Catholique, CSPS de Gogo).

\subsubsection{Agents de santé}

Les agents de santé sont les personnes spécialisées indiquées et habilitées à offrir les soins à la population en général et aux adolescentes en particulier. Leurs rôles dans la gestion de la grossesse des adolescentes sont de :

- assurer la CPN,

- apporter les soins médicaux aux adolescentes en grossesse,

- assurer la prise en charge psycho sociale des adolescentes en grossesse,

- assurer l'accouchement,

- apporter les soins aux nouveau-nés et aux post-partum.

Tous ces acteurs jouent des rôles complémentaires et visent les mêmes objectifs à savoir assurer le bon déroulement de la grossesse jusqu'à son terme. La cellule familiale se base davantage sur son expérience et les pratiques traditionnelles pour apporter sa contribution au bon déroulement de la grossesse tandis que les agents de santé apportent les soins modernes plus adaptés. 
Cependant, il convient de relever l'absence de collaboration entre les deux niveaux. Il s'avère donc nécessaire d'établir et de renforcer la collaboration afin de réduire les contradictions entre les une et les autres et faire en sorte que le niveau familial s'améliore dans la prise en charge de la grossesse chez les adolescentes.

La gestion des grossesses implique aussi des prises de décisions relatives au suivi et aux soins liés à la grossesse. L'étude a nettement fait ressortir les différents niveaux de prise de décision qui varient d'un groupe ethnique à un autre.

Chez les Mossi, c'est le mari de l'adolescente qui est le décideur principal ; la femme appartient d'abord à son mari et par conséquent, il est le seul habilité à décider du suivi médical et des soins de sa femme. Néanmoins, ses parents participent à la prise de cette décision.

Pour les Peulh, les Gourmantché et les Bissa, c’est la belle-mère qui prend les décisions relatives au suivi et soins médicaux de sa belle-fille. Chez les Peul particulièrement, le pouvoir de décision de la belle-mère dans la gestion de la grossesse est plus important car le mari n’intervient presque pas. Néanmoins, elle sollicite souvent l’avis du beau-père.

\subsubsection{Appréciation des prestations des services de santé par les adolescentes}

La majorité des adolescentes enquêtées apprécient positivement la qualité de prestations des agents de santé lors du suivi de la grossesse, durant l'accouchement et pendant la période postpartum.

Ces appréciations portent essentiellement sur l'accueil, les conseils et les soins reçus.

Cette majorité reconnaît avoir été bien reçue par les agents de santé qui l'ont mise en confiance et à l'aise lors de la première CPN.

Les adolescentes reconnaissent également avoir reçu des conseils de l'accoucheuse auxiliare et/ou de la sage-femme lors de la période de la grossesse, durant l'accouchement et pendant la période post-partum. Ce sont :

- la conduite à tenir face à une grossesse

- la manière de se coucher avec la grossesse

- le respect des restrictions vestimentaires

- l'observation des mesures d’hygiène corporelle durant la grossesse

- l'hygiène alimentaire

- l'allègement des charges de travail

- Le recours à un centre de santé pour le suivi de la grossesse et l'accouchement

- le recours à l'AV en cas de problèmes

- l'utilisation d'un lavement évacuateur avant l'accouchement

- le matériel à apporter pour l'accouchement

- le respect de la consultation post-natal et de la vaccination du nouveau-né

- l'allaitement exclusif au sein du nourrisson

- l'hygiène du nourrisson

- les signes de danger pour l'adolescente et le nourrisson durant la période post-partum 
- l'utilisation des contraceptifs à la période post-partum

- Etc.

Quant aux soins reçus, ils concernent essentiellement: les prescriptions médicales lors des malaises et/ou maladies, les soins apportés à la post-partum et au nouveau-né.

« J'ai reçu des conseils au CSPS sur comment se conduire avec la grossesse » (Adolescente post-partum, 18 ans, Mossi, catholique/Kaya).

"Dès que je me sens mal et que je vais au CSPS, j'ai les soins qu'il faut.» (Adolescente en grossesse, 17 ans, Mossi, catholique/Manga).

"Depuis ma grossesse jusqu'à maintenant, J'ai eu les soins nécessaires chaque fois que je me suis rendue au CSPS » (Adolescente post-partum, 19 ans, Catholique, mossi/Gogo).

Seules les adolescentes en grossesse et post-partum rencontrées à Bogandé ne sont pas satisfaites des prestations de services des agents de santé. Les raisons évoquées sont : le mauvais accueil, les mauvais traitements verbaux, la négligence, la non assistance aux femmes en couche.

« Dès fois, les accoucheuses sont absentes. Le jour où j'ai accouché, elle dormait, elle n'a pas voulu se réveiller et elle m'a laissée accoucher toute seule (Focus, adolescente post-partum, Bogandé).

"Le jour où j'ai accouché, l'accoucheuse dit qu'elle est fatiguée et c'est ma mère qui m'a accouchée au centre de santé » (Focus, ados post-partum, Bogandé).

« Je vais à la CPN, mais ma tension n'a pas été prise car il paraît que dans le nouveau carnet, il n'y a pas de place pour noter les tensions » (Focus, adolescente en grossesse, Bogandé).

\subsubsection{Besoins des adolescentes en matière de soins pendant la grossesse}

D’une manière générale, les adolescentes ont exprimé des besoins en matière de soins pendant la grossesse. Ces besoins portent sur l'entretien d'une grossesse, la contraception, l'alimentation pendant la grossesse, les comportements et attitudes à adopter lors de la grossesse, les soins pour assurer le bon développement du fœtus et d'autres types d'informations diverses sur la grossesse. Pour supporter les frais des soins inhérents à la grossesse, les adolescentes ont souhaité des appuis à la création d'AGR. 


\subsubsection{Capacités des services de santé à répondre aux besoins des adolescentes}

Sur l'ensemble des douze sites retenus, un seul village (Tibilindi) n'a pas de structure sanitaire. Aussi, sa population doit se déplacer à Gangaol, situé à trois (03) kilomètres pour y recevoir les soins.

Toutes les structures sanitaires offrent les mêmes types de prestations. Il a été constaté l'absence de prestations spécifiques pour les adolescentes en grossesse et post-partum. Les CSPS qui constituent l'essentiel de notre élément d'investigation ont tous la même configuration en termes de services et de personnels, à quelques exceptions près. Selon les normes, le personnel comprend : un infirmier d'Etat, et/ou breveté, une accoucheuse auxiliaire, un agent itinérant de santé. Mais, en plus de ce personnel de base, on a rencontré des sages-femmes travaillant dans les CSPS urbains de Dori, Kaya, Tenkodogo, Manga. En dépit de cet effectif réduit, les agents de santé arrivent à répondre aux besoins thérapeutiques des adolescentes mariées en grossesse, pour l'accouchement et la période post-partum car elles ne sont pas traitées différemment des femmes adultes qui sont dans la même situation. Mais, ils n'arrivent pas à prendre en charge les besoins spécifiques de ces adolescentes qui exigent beaucoup plus d'attentions.

Il n'en est pas de même pour les adolescentes non mariées en grossesse ou non qui n'ont généralement recours aux centres de santé qu'en dernier ressort, lorsqu'elles sont au bord du désespoir. L'insuffisance d'agents fait que la prise en charge de ce groupe n'est assurée que partiellement. En effet, même pour les agents qui ont été formés dans la prise en charge des adolescentes, il leur est difficile d'assurer correctement cette prestation spécifique qui exige un counseling pour les mettre d'abord en confiance avant de faire la consultation.

"Il faut une attention particulière pour les adolescentes. Le jour où j'ai 2 adolescentes, je ne peux plus en consulter d'autres, cela me prend énormément de temps car il faut trouver les mots justes étant donné que la grossesse est une surprise pour elle. » (Accoucheuse Auxiliaire, CSPS urbain, Tenkodogo).

«Il faut une formation spécifique pour gérer la grossesse des adolescentes non mariées. Dès que tu rates le départ où qu'elles ne sont pas satisfaites, elles ne reviennent plus et le plus souvent vont avorter. » (Sage-femme, CSPS urbain, Dori).

Par ailleurs, l'absence d’espace aménagé pour les adolescentes dans les centres de santé réduit aussi la capacité des centres de santé à répondre à leurs besoins. Ces dernières utilisent la même salle d'attente que les adultes et sont consultées dans la même salle ; ce qui constitue une gêne pour elles. Aussi, une réorganisation des services de santé, un renforcement des capacités techniques sont nécessaires en vue d'améliorer la prise en charge totale des adolescentes.

\subsection{Itinéraires thérapeutiques des adolescentes pour l’accouchement}

L'accouchement est l'aboutissement naturel de la grossesse. A ce titre, il apparaît également comme un phénomène social valorisant comme le révèle l'analyse de la littérature sur le sujet. En effet, des auteurs comme OUEDRAOGO Koumbou affirment que la maternité est «l'assurance 
de la prospérité de la famille » et LALLEMAND Suzanne de renchérir qu'elle «valorise qui la donne ».

\subsubsection{Itinéraires thérapeutiques}

L’objet de cette partie est tout d'abord de présenter une typologie des trajectoires suivies (itinéraires thérapeutiques) par les enquêtées à partir du début du travail jusqu'à l'accouchement. Ensuite, il s'agira d'analyser les conditions d'accès au centre de santé qui seront appréhendées à travers les parcours des adolescentes.

$\mathrm{Au}$ terme de la grossesse, dès que les adolescentes constatent les signes précurseurs de l'accouchement, la majorité d'entre elles est conduite au centre de santé par les belles-mères et/ou les femmes âgées de la famille pour y accoucher. Cette étape est gérée exclusivement par les agents de santé qui leur donnent les soins adéquats.

Si le travail commence, on donne à boire à l'adolescente le "Nassidji ${ }^{11}$ » ou/et un médicament à purger pour faciliter l'accouchement. Si le travail se déroule normalement, elle accouche à la maison. C'est seulement en cas de problèmes qu'elle est conduite au centre de santé. Il en est de même des belles-mères qui sont aussi favorables à l'accouchement au centre de santé.

"Elle va accoucher au CSPS car s'il y a un problème au niveau de la mère et du bébé, les agents de santé peuvent prendre les mesures nécessaires. » (Mari d'adolescente en grossesse, 24 ans, Malébé, musulman/Saouga).

"Elle va accoucher au CSPS car il y a l'accoucheuse ; les médicament aussi sont disponibles en cas de problèmes... » (Mari d'adolescente en grossesse, 28 ans, Mossi, musulman/Noaka).

«Ma deuxième belle-fille va accoucher au CSPS comme l'autre. C'est moi qui l'ai décidé car accoucher à la maison est très risquant... » (Belle-mère d'adolescente en grossesse et postpartum, 49ans, Malébé, musulmane/Saouga).

En résumé, on peut retenir que l'itinéraire de l'accouchement de la quasi-totalité des adolescentes en grossesse dans les différentes zones de l'étude est le recours à un centre de santé. Ce comportement se justifie par la présence de motivations telles que les soins obstétricaux d'urgence, et aussi par l'amélioration de la qualité des prestations des agents de santé et de l'accessibilité géographique d'un centre de santé.

«Le taux d'accouchement assisté est assez satisfaisant en ce moment grâce au SONU, il est de 60\%. » (Accoucheuse auxiliaire, CSPS, Bissiga).

«Le projet Help qui prend en charge les femmes enceintes, les post-partum et les enfants de 0 à 5 ans, a fait croître le taux de fréquentation de la maternité; et c'est là où beaucoup d'adolescentes viennent pour la CPN... » (Agent de santé, CSPS urbain, Dori.).

\footnotetext{
${ }^{11}$ Substance liquide dotée de pouvoirs mystiques fabriquée par les marabouts
} 


\subsubsection{Connaissances sur l'accouchement}

La plupart des adolescentes enquêtées n’ont pas d'informations sur l'accouchement pour des raisons liées à la peur, à la pudeur, à l'ignorance des sources d'informations. L'accouchement apparaît chez les adolescentes comme une expérience à vivre et non à raconter. Leur niveau d'information sera analysé à travers leur connaissance des signes d'une grossesse à terme.

Pour les adolescentes, l'âge de la grossesse est le premier indicateur pour reconnaître que la grossesse est à terme. Cependant, il se pose un problème, car dans la plupart des cas, elles ne maîtrisent pas le mode de calcul pour connaître l’âge de la grossesse.

Les signes de l'imminence de l'accouchement sont plus connus parce que se manifestant généralement par des douleurs. En effet, selon la majorité des adolescentes post-partum enquêtée, les maux de bas-ventre constituent le signe annonciateur majeur de l'imminence de l'accouchement. Ce signe a été notifié par l'agent de santé aux adolescentes et par tous les autres acteurs intervenant dans la gestion de l'accouchement. Cette accoucheuse affirme insister sur cet aspect en ces termes: "Dès que vous avez des maux de bas-ventre qui persistent, venez à la maternité... » (Accoucheuse/Saouga/Oudalan.)

Les belles-mères connaissent ce signe et l'ont également évoqué.

\section{«Elle a dit qu'elle a mal au ventre et quand je l'ai vu, j'ai su qu'elle était à terme. (Belle-mère} d'adolescente post-partum/ Tamashek/Musulmane/Dori).

De façon générale, les AV ont de bonnes connaissances sur les signes d'une grossesse à terme grâce aux formations reçues. Les signes cités sont entre autres la position basse du ventre. En revanche, selon elles, la connaissance de l'imminence de l'accouchement de l'adolescente est liée à leur expérience professionnelle. Elles procèdent également à un examen pour confirmation comme l'affirment ces AV :

" Lorsque le ventre descend plus bas, c'est qu'il est à terme» (AV, Bissiga).

«Si la fille vient et que je la consulte, si c'est le moment d'accoucher, je le sens tout de suite. » (AV, Gogo).

Quant aux signes annonciateurs d'un accouchement difficile, la quasi-totalité des adolescentes affirme ne pas les connaître. Il en est de même pour les belles-mères dont la majorité affirme n'avoir pas les compétences requises pour identifier les signes d'un accouchement qui va se dérouler difficilement.

La minorité des belles-mères qui a pu donner des signes a évoqué la longue durée du temps de travail et la hauteur du ventre.

Selon elles, quand un accouchement dure plus d'une journée, c'est qu'il y a un problème. Elles estiment aussi que la position du ventre bas augure d'un bon accouchement et lorsqu'il reste assez haut, c'est que l'accouchement sera difficile :

"Quand le travail dure plus de 24 heures, on dit que l'accouchement est difficile » (Belle-mère adolescente post-partum Bella/Musulmane/Dori). 
"Si l'accouchement est facile, le bébé descend, alors que si c'est difficile, le ventre reste en haut... », (Belle-mère adolescente post-partum Malébé/Musulmane/Saouga).

Seules les hémorragies et la présentation du bébé ont été évoquées comme signes d'accouchement difficile par les AV qui accouchent.

"C'est lorsque la femme commence à saigner au moment du travail ... ou si l'enfant se présente par le pied ou la main qu'on peut dire que l'accouchement va être difficile», (Accoucheuse villageoise, Torodi/ Dori).

«Avant l'accouchement, si je constate qu'au toucher, la dilatation n'avance pas, c'est qu'il y a problème, je fais appel à l'accoucheuse...", (Accoucheuse villageoise, Mossi, Catholique, Noaka).

Il ressort de ces informations que les acteurs communautaires ont de faibles connaissances sur l'accouchement. La majorité d'entre eux pense que l'accouchement est une question technique qui doit exclusivement être traitée par les agents de santé. Même si les signes inquiétants ne sont pas connus de la majorité, tous sont unanimes à reconnaître qu'en cas d'alerte, il faut conduire l'adolescente au centre de santé pour une meilleure prise en charge.

\subsubsection{Vécu de l’accouchement}

L'accouchement est un processus qui comprend principalement deux phases à savoir une étape préparatoire et celle de l'accouchement proprement dit.

L'étape préparatoire concerne toutes les dispositions à prendre sur le plan matériel et financier pour un bon déroulement de l'accouchement. La préparation de cette étape varie selon les groupes ethniques. Si les Peulh accordent une importance particulière à cette phase, on note une insuffisance dans la préparation matérielle chez les Mossi et les Gourmantché, à cause de croyances traditionnelles selon lesquelles la préparation à l'avance d'un accouchement peut entraîner la mort du bébé et/ou de la mère. Ainsi, les achats (vêtements, savon, etc.) sont faits seulement à la naissance du bébé. Les propos suivants recueillis à Bissiga mettent en évidence un aspect de ces croyances :

«Ma belle-mère m'a dit de ne rien préparer car ce n'est pas bien. Elle a dit que si j'achète des affaires, je vais accoucher d'un mort-né», (Adolescente en grossesse, Mossi, Catholique/ Bissiga).

Cependant, certaines affirment prendre les dispositions requises. Généralement, ce sont les belles-mères et les maris qui se "préparent » ou qui préparent l'accouchement en faisant de l'épargne. Des habits, du savon, des berceaux et autres sont achetés pour la circonstance comme le disent ces deux belles-mères :

"J'achète des habits pour le bébé, du savon et quelques autres nécessaires à l'approche de l'accouchement » (Belle-mère adolescente en grossesse Bella, Musulmane/Dori). 


\section{" J'achète le berceau, les habits, du savon et j'épargne 5000F » (Belle-mère adolescente post- partum Mossi, Catholique/Manga).}

La grande majorité des hommes affirment tout mettre en œuvre pour le bon déroulement de l'accouchement de leurs épouses à travers le suivi médical de la grossesse et l'allègement des tâches. De plus, les maris des adolescentes en grossesse ont déclaré qu'ils amèneront (pour les maris d'adolescentes en grossesse) ou qu'ils ont déjà amené (pour les maris d'adolescentes postpartum) leurs épouses dans un centre de santé pour une meilleure prise en charge de l'accouchement comme l'affirme ce mari.

"Elle a accouché au CSPS parce qu'en cas de problèmes au niveau du bébé ou de la mère, ils peuvent prendre les mesures nécessaires. » (Mari d'adolescente post-partum, 24 ans, Malébé, musulman /Saouga).

La prévision est surtout faite pour ce qui est des personnes accompagnatrices. La belle mère, une coépouse ou une vieille de la concession identifiée à l'avance pour accompagner la fille au centre de santé dès l'apparition des premiers signes. Les moyens de transport pour l'occasion sont constitués de vélos, de motocyclettes et même de charrettes selon les moyens et les possibilités. Certains y vont à pieds quand aucun moyen n'est disponible.

Au moment de l'accouchement proprement dit, la majorité des adolescentes et des autres acteurs reconnaissent ne pas faire de préparation spécifique car selon eux, cette phase est sous contrôle exclusif des agents de santé du fait qu'il se passe au centre de santé. Leur contribution à ce stade se résume aux prières.

Mais chez les Peulhs, au-delà de la préparation matérielle, l'adolescente est éduquée à supporter la douleur de toute nature sans aucune manifestation extérieure, y compris celle de l'accouchement. La femme en général et l'adolescente en particulier subit une éducation et une pression sociale de sorte à ne pas extérioriser les douleurs et celles liées à l'accouchement. Tous cris ou pleurs de la part de l'adolescente constituent une honte pour sa famille d'origine. Cette accoucheuse du CSPS de Saouga témoigne en ces termes :

"Ici, à l'accouchement, les femmes, quel que soit l'âge, ne doivent jamais crier, ni exprimer leur douleur car ce sera la honte de la famille. Il faut avaler la douleur et même si tu dois mourir, tu pars avec la douleur. On leur donne un chiffon afin qu'elles mordent dedans pour ne pas crier » (Accoucheuse auxiliaire, CSPS Saouga).

Les agents de santé, quant à eux, mettent plutôt l'accent sur les risques encourus par les adolescentes pendant le vécu de leur accouchement. Au regard de leur âge précoce et du fait qu'elles sont en général des primipares, elles présentent souvent plus de risques de complication à l'accouchement.

Les agents de santé soulignent d'autres risques supplémentaires si l'adolescente accouche à domicile comme c'est le cas dans certains villages éloignés des centres de santé. Les risques encourus évoqués par les agents de santé sont entre autres les déchirures, les risques de décès du bébé et la fustile à long terme comme le confirment ces propos. 
"Toute femme en grossesse est sujet à une complication quel que soit l'âge. Elle court plus de dangers quand c'est une adolescente. Même avec la CPN, il peut y avoir des problèmes à l'accouchement dont la fistule, entre autre ", (Sage-femme, Dori).

"Il y a une déchirure si l'adolescente accouche à domicile ; il y a aussi une souffrance foetale quand le bassin de la fille est petite, et quelques rares fois de mort-nés lorsque le travail dure près de 2 jours », (Accoucheuse auxiliaire, Saouga).

\subsubsection{Rituels, restrictions et conseils lies à l'accouchement}

Il y a très peu de rituels liés à l'accouchement sauf quelques rares consultations de devins suivies de sacrifices et d'aumônes divers dont le but est de faciliter l'accouchement. Ces pratiques sont présentes à des degrés différents dans toutes les communautés visitées.

Chez les Peulh, pour faciliter l'accouchement, il existe une pratique qui consiste à badigeonner le ventre de l'adolescente avec du beurre de vache avant de la transporter au centre de santé comme le montrent les propos de cette belle-mère rencontrée à Tibilindi :

«On frotte du beurre de vache sur le ventre de l'adolescente au moment du travail en vue de faciliter l'accouchement » (Belle-mère d'adolescente post-partum, Peulh, Tibilindi/Séno).

Certaines considérations socio-culturelles peuvent limiter l'accouchement des adolescentes au centre de santé. Dans la Gnagna par exemple, si un centre est situé à l'Est, la femme enceinte ne doit pas s'y rendre parce qu'elle ne doit pas aller dans cette direction. Il arrive souvent qu'il n'existe pas de centre de santé dans la direction souhaitée. Cette situation peut entraîner des risques suplementaires de danger pour l'accouchement des adolescentes. C'est ce que dit cette accoucheuse rencontrée dans le village de Gnongboudeni à la périphérie de Bogandé :

"Les adolescentes n'accouchent pas à la maternité mais elles y viennent pour le suivi de la grossesse. Elles n'y accouchent pas car c'est le guérisseur traditionnel qui doit indiquer le lieu de l'accouchement... ». (Accoucheuse auxiliaire, CSPS de Gnongboudéni, Bogandé).

\subsubsection{Profils et rôles des personnes ressources dans la gestion de l’accouchement}

Plusieurs personnes sont impliquées dans la gestion des accouchements des adolescentes. Ces personnes interviennent selon le stade d'évolution de l'accouchement.

Le premier stade qui est celui de la préparation matérielle et financière incombe généralement au mari et à la belle-mère pour les adolescentes mariées et la famille d'origine pour celles non mariées. Chez les Peul, cette responsabilité incombe souvent à la tante et/ou la mère de l'adolescente.

Pour l'accompagnement de l'adolescente à aller accoucher, plusieurs personnes sont également impliquées. Il s'agit principalement de la belle-mère. Mais il ressort de nos enquêtes que d'autres personnes âgées de la belle-famille ou une coépouse ayant déjà une expérience de l'enfantement 
peut aussi jouer ce rôle. En réalité, toute femme est habilitée à l'accompagnement si elle est la seule disponible au début du travail de l'adolescente :

«Ce n'est pas obligé que la belle-mère accompagne la fille au CSPS, d'autres femmes de la cour ou des coépouses peuvent le faire aussi » (Belle-mère adolescente post-partum peul/

musulmane/ Tibilédi).

Le mari intervient dans l'accompagnement en transportant la parturiente jusqu'au lieu de l'accouchement et en réglant les différents frais y afférents. Sa présence sur les lieux de l'accouchement n'est d'ailleurs pas souhaitée par les adolescentes. Elles affirment que leur mari pourrait entendre leurs cris et se moquer d'elles. Pour d'autres également aussi, l'accouchement n’est pas un problème géré par les hommes, mais par les femmes uniquement.

La belle-mère est également chargée d'assister l'adolescente en couche, exception faite chez les Peulhs où l'adolescente rejoint sa famille d'origine à partir du septième mois de la grossesse. Elle ne retourne dans sa belle-famille que quelques mois (3 à 4 mois environ) après son accouchement.

Pendant l'accouchement, on note souvent la présence d'autres femmes de la concession (coépouses, sœurs, femmes des frères du mari, etc.) qui assistent souvent la belle-mère dans le nettoyage de la salle d'accouchement, la recherche d'eau et l'exécution de petites commissions diverses. Elles apportent également un soutien moral à l'adolescente après son accouchement.

Les belles-mères affirment cependant ne pas connaître le type de conseils à donner à l'adolescente au moment du travail sauf que de prier. Les maris également ont affirmé s'adonner à la prière pendant l'accouchement de leur femme. C'est ce qui ressort des propos suivants de belles-mères et de maris :

«Quand j'ai su que ma belle-fille était en travail, je ne savais que dire, ni que faire, je n'ai fait que prier ", (Belle-mère d'adolescente post-partum, Malébé/Musulmane/Saouga).

"J'attendais à la maison, j'ai prié Dieu pour qu'elle accouche en paix.», (Mari adolescente post-partum, 24 ans, Malébé, Musulman/Saouga).

Le choix du lieu de l'accouchement est fait dès la découverte de la grossesse par la belle-mère et le mari de l'adolescente. Par ailleurs, la quasi-totalité des adolescentes post-partum, déclare avoir accouché dans un centre de santé et que ce choix leur a été suggéré par les agents de santé lors du suivi de la grossesse. Pour elles, si la CPN est faite au centre de santé, il n'y a pas de raison qu'elles n'y accouchent pas. Ainsi, il ressort de nos données que les adolescentes qui ont fait la CPN s'orientent plus facilement au centre de santé pour les accouchements.

Dans les différentes zones de l'étude, le recours à un centre de santé pour l'accouchement se justifie par la qualité des prestations et à son accessibilité géographique. La gratuité et/ou la réduction des actes pour les accouchements au centre de santé est un facteur incitateur supplémentaire pour les adolescentes, surtout celles non mariées, de recourir au centre de santé. En effet nos enquêtes ont révélé un système de motivation des femmes enceintes et post-partum 
dans certains CSPS (CSPS de Gnongboudeni) à travers la distribution gratuite de moustiquaires imprégnés et de vivres aux post-partum.

C'est le cas également du projet HELP qui appuie le CSPS urbain de Dori dans la prise en charge des frais liés au suivi médicale des femmes enceintes, des femmes allaitantes et des enfants âgés de 0 à 5 ans. C'est ce qu'affirment les propos de cet agent de santé :

Le projet Help qui prend en charge les femmes enceintes, les femmes allaitantes et les enfants de 0 à 5 ans a fait croître le taux de fréquentation du CSPS et c'est là où beaucoup d'adolescentes viennent consulter ». (Agent de santé, CSPS urbain Dori).

Dans la majorité des cas, les belles-mères et les maris affirment être favorables à l'accouchement assisté en milieu sanitaire. Ils disent participer et même influer sur le choix du recours au centre de santé pour l'accouchement des adolescentes. L'adhésion au choix du centre de santé pour l'accouchement est liée surtout à la qualité de la prise en charge médicale de celui-ci comme l'attestent les propos suivants :

«Elle va accoucher au CSPS car s'il y a un problème au niveau de la mère ou du bébé, ils peuvent prendre les mesures nécessaires...» (Mari d'adolescente en grossesse/Malébé/Musulman/Saouga).

"Ma 2ème belle-fille va accoucher au CSPS .comme l'autre. C'est moi qui l'ai décidé ainsi car rester à la maison est très risquant. Et puis, si tu accouches à la maison et que tu te présentes après au CSPS en cas de problème, les agents de santé te négligent... » (Belle-mère adolescente en grossesse et Post-partum/Malébé/Musulmane, Saouga).

«Elle va accoucher au CSPS car il y a l'accoucheuse et les médicaments sont disponibles en cas de problèmes... » (Mari d'adolescente en grossesse/Mossi/Musulman/Noaka).

Même si la majorité des adolescentes déclare accoucher au cantre de santé, on a noté quelques cas d'accouchements et des intentions d'accouchement à domicile. Il ne s'agit pas d'un choix délibéré de la part de celles-ci, mais cela est dû aux facteurs suivants :

- le manque d'argent ;

- l'ignorance ;

- l'éloignement des centres de santé ;

- la méconnaissance de l'imminence de l'accouchement.

Cette adolescente justifie son accouchement à domicile par sa méconnaissance de l'imminence de son accouchement :

« J'ai accouché à la maison. Ce n'était pas de ma faute. J'ai pris la bouilloire pour aller pisser seulement et l'enfant est tombé » confie une ado à l'accoucheuse (Adolescente post-partum /peulh/ Musulmane/ Saouga). 


\subsection{Itinéraires thérapeutiques des adolescentes pendant la période post-partum}

La période du post-partum s'étend de la fin de l'accouchement jusqu'au retour des couches, c'està-dire les premières règles après la grossesse. C'est une période de nouveaux bouleversements à la fois psychiques que familiaux (période clef pour la mise en place de la relation mère-enfant, de la découverte du nouveau-né, de mutations familiales), mais aussi physiques avec la perte brutale des repères physiologiques et anatomiques liés à la grossesse. Le post-partum est donc une période à risque faite de difficultés, parfois de complications, liées aux bouleversements de tous les repères d'une femme en particulier lorsqu'il s'agit de son premier enfant. Celle-ci mérite pour ces raisons un suivi et une attention particulière. L'adolescente bien encadrée et entourée est parfaitement capable d'une attitude parentale tout à fait correcte.

\subsubsection{Itinéraires thérapeutiques}

Après avoir reçu les soins donnés par les agents de santé à la maternité, les adolescentes et leurs nourrissons reçoivent d'autres soins à base de plantes médicinales à domicile quelle que soit la région visitée et les ethnies considérées. Ces soins sont généralement prodigués par les bellesmères, mères ou autres femmes de la famille dans le but de rendre le bébé vigoureux et d'aider la mère à retrouver une bonne santé.

Même si la grande majorité des adolescentes post-partum affirment ne pas connaître des signes évocateurs des problèmes de santé chez la mère et l'enfant, elles ont recours à l'automédication à travers l'utilisation des décoctions. Le recours au centre de santé est l'étape suivante s'il n'y a pas d'amélioration. Parfois, une association des soins traditionnels et modernes est faite en même temps.

Les résultats de l'étude montrent cependant que, pour certains types de soins tels les vaccins, les consultations, la planification familiale, les adolescentes retournent toujours au centre de santé après l'accouchement.

En dehors de l'automédication et du centre de santé, certaines adolescentes post-partum ont recours aux guérisseurs traditionnels. Ils aident également les post-partum pour les maux de ventre, les ballonnements, et aussi pour avoir du lait. Ils s'occupent de la santé des nourrissons par l'administration de soins à base de plantes médicinales et autres mixtures. En plus des soins, les guérisseurs donnent des conseils aux parturientes sur l'alimentation, l'entretien et l'hygiène du nourrisson.

"... Les post-partum viennent avec leurs nourrissons qui font de la fièvre, elles prennent des décoctions en vue d'avoir du lait... . (Guérisseur, 47ans, Mossi, Chrétien, Bissiga).

" Je donne des conseils aux post-partum en leur disant de faire bouillir des décoctions à boire, à laver pour faciliter le rejet des déchets ; laver l'enfant avec l'eau chaude et nettoyer les seins avant de les donner au bébé.» (Femmes Guérisseur, 43 ans, Gourmantché, musulmane/Bogandé). 


\subsubsection{Prise en charge de l’adolescente durant la période post-partum}

\subsubsection{Nature et qualité des informations/soins et conseils reçus par l'adolescente}

Après l'accouchement, toute l'attention est portée au nouveau-né et à la mère. L'adolescente découvre au fur et à mesure les effets de l'accouchement. Des soins modernes et traditionnels leurs sont administrés.

Pour les soins modernes, l'adolescente reçoit un sérum antitétanique et des médicaments qui facilitent la reconstitution rapide de l’utérus.

Quant aux soins traditionnels, ils portent sur la toilette intime de l'adolescente afin qu'elle recouvre le plus vite possible sa force et la correction au niveau de son bas-ventre. Seules les douches chaudes avec des décoctions ont été signalées comme pratiques traditionnelles liées au post-partum pour l'adolescente.

"On m'a lavé à l'eau chaude et masser mon ventre avec du beurre de karité après l'accouchement, on a lavé aussi le bébé avec des décoctions » (Adolescente post-partum, 19 ans, musulmane, Mossi, Bissiga).

En dehors de ce type de soins, des restrictions alimentaires lui sont dictées par rapport à la consommation d'aliments tels que les aliments amers, le miel, le lait, les fruits, le concombre qui peuvent altérer la qualité du lait. Les aliments recommandés à la mère à savoir le «zoomkoom $^{12}$ » et la bouillie, favorisent la sécrétion du lait.

Durant la période post-partum, certaines adolescentes utilisent le «nassidji » donné par des marabouts pour se protéger des maladies qui surviennent après les suites de couche.

\subsubsection{Soins au nouveau-né}

Le nouveau-né reçoit deux types de soins pour la majorité des adolescentes. Il s’agit des soins modernes et des soins traditionnels. Pour les soins traditionnels, il s'agit, selon les belles- mères, de l'utilisation de l'eau chaude pour la toilette et du beurre de karité pour le massage du corps. Certaines d'entre elles reconnaissent cependant recourir à des décoctions pour laver et purger le bébé pour lui rendre sa vitalité et sa vigueur en vue de prévenir les maladies.

Les soins modernes concernent surtout le recours aux centres de santé pour les pesées, les vaccins et les maladies. La majorité des adolescentes affirment assurer le suivi médical de leurs bébés à travers la pesée au centre de santé. Aussi, en terme de fréquence dans la fréquentation du centre de santé, les adolescentes post-partum disent y retourner au moins trois fois : une semaine après l'accouchement pour le vaccin du bébé, une deuxième fois pour la consultation relative aux suites de couches et enfin la troisième pour la PF.

En dehors de ce parcours thérapeutique, des pratiques sont usitées par les belles-mères à travers l'utilisation d'amulette ou de « nassidji » pour protéger le nouveau-né du « mauvais œil ».

\footnotetext{
${ }^{12}$ Boisson farineuse sucrée à base de petit mil
} 
«Dès la sortie de la maternité, je suis allée voir le marabout pour avoir quelque chose pour la protection du bébé : une amulette à attacher à ses reins et du "nassidji » pour le laver» (Bellemère adolescente post-partum, 35 ans, Peulh musulmane/Tibilindi).

Un nombre important de belles-mères mentionne des cas de maladies chez les nouveau-nés. Dans la majorité des cas, la médecine traditionnelle est le premier recours thérapeutique et la médecine moderne, le second ou encore une association des deux.

«Deux semaines après sa sortie de la maternité, le bébé a fait de la fièvre ; je l'ai soigné avec des décoctions, mais comme la fièvre persistait, on l'a amené au CSPS » (Belle-mère ado postpartum, Mossi, Animiste/Noaka).

En plus des soins, des conseils sont donnés par l'agent de santé sur les soins à apporter au nouveau-né. Ils portent généralement sur :

- les bonnes manières pour prendre un bébé

- l'alimentation riche pour favoriser la venue du lait

- l'hygiène du bébé

- l’hygiène des tétons avant la tétée

- etc.

« Je lui ai appris comment donner à téter au bébé, je lui ai dit d'observer l'hygiène du bébé, de bien protéger le bébé contre le froid, de bien le tenir pour ne pas le blesser, de bien manger pour avoir beaucoup de lait. » (Belle-mère adolescente post-partum, 30ans, Catholique, Bobo, Tenkodogo).

L'apprentissage des soins se fait donc au fur et à mesure de la croissance du bébé. La majorité des adolescentes interrogées ne disposaient pas d'informations sur les soins du bébé avant l'accouchement et n'en ont pas recherchées. Pour d'autres, en plus des mères ou des bellesmères, les sages-femmes sont également des sources d'informations sur les soins aux nourrissons. Elles affirment recevoir ces informations lors des séances de pesée ou de sensibilisation au niveau des centres de santé.

\subsection{Planification familiale}

Si avant la grossesse, la contraception n'est pas envisagée par l'adolescente qui vient de se marier, elle l'est chez la post-partum. La majorité des adolescentes en grossesse et post-partum enquêtées affirme connaître des méthodes contraceptives par le biais des agents de santé lors des CPN ou des pesées des nourrissons. Elles en ont également entendu parler lors des sensibilisations faites à travers la radio, la télévision, le théâtre, les cinés-débats, les cours ou encore à travers les échanges entre copines.

Tout comme leurs maris, elles ont entendu parler de la pilule, du norplant, du préservatif, des injectables, du collier, du stérilet et de l'abstinence. La majorité des post-partum utilisent les injectables et le norplant. 
La plupart des adolescentes sont favorables à l'utilisation de moyens contraceptifs au vu des conditions de vie de plus en plus difficiles et précaires. Leur préférence pour l'espacement des naissances va de 3 à 4 ans.

Il faut noter qu'une minorité de maris d'adolescentes reste toujours réticente du fait des rumeurs qui disent que la prise de pilules ou les injections rendent les femmes stériles ou entraînent des accouchements difficiles.

« Je ne laisse pas ma femme utiliser les contraceptifs parce que les comprimés entraînent des difficultés à l'accouchement; accouchement difficile avec opération. " (Mari adolescente 32 ans, Mossi, musulman/Bissiga).

«Avec sa femme, il n'est pas nécessaire d'utiliser une méthode contraceptive. C'est plutôt bien pour les maîtresses dehors» (Mari adolescente en grossesse, 28 ans, Songhaï, musulman/Saouga).

De nos jours, les mentalités semblent de plus en plus tolérantes et la majorité des belles-mères interrogées sont favorables à l'utilisation des contraceptifs par leurs filles ou belles-filles.

" Je vais dire à mon fils et à sa femme d'aller au CSPS pour faire la piqûre en vue d'espacer les naissances ...» (Belle-mère d'adolescente post-partum, 49 ans, Malébé, musulmane, Saouga/Oudalan).

«Pour que ma belle-fille puisse attendre 3 ou 4 ans sans accoucher, je lui ai dit d'aller au CSPS demander de l'aide ». (Belle-mère adolescente post-partum, 38 ans, Peulh, Musulmane/Tibilindi).

La première méthode envisagée aussi bien chez les adolescentes que chez leurs maris est l'abstinence. Elle est la méthode la plus connue et la plus courante pendant l'allaitement du bébé. La stratégie consiste à un séjour de la femme chez ses parents où qu'elle aménage avec sa bellemère et ce, jusqu'à ce que l'enfant fasse ses premiers pas. Cependant la majorité des adolescentes bien qu'elles citent d'emblée l'abstinence comme méthode, restent conscientes de la difficulté de l'observance de cette méthode.

« Après la maternité, la fille est dans la concession de sa belle-mère durant 3 à 4 années avant de rejoindre son mari. Le bébé aura grandi pendant ce temps. » (Belle-mère d'adolescente postpartum/Gourmantché, Musulmane/Manni).

La deuxième méthode répandue est l'injectable qui est connue, citée et utilisée par la majorité des post-partum.

Le choix de la méthode de planification des naissances doit faire l'objet d'un choix concerté entre les conjoints. Mais, selon les informations collectées, rares sont les adolescentes qui ont échangé sur cette question avec leurs conjoints ou ont pris cette décision librement. Toutefois, la réticence vient surtout des hommes à cause du fait des pesanteurs socioculturelles. Le centre de décision reste souvent entre la belle-mère et le mari de l'adolescente. Quelques fois, la religion est un élément décisif pour son adoption ou non. Certaines d'entre elles et leurs conjoints souhaitent avoir des informations sur les méthodes contraceptives et leur mode d'utilisation. 

Les résultats de l'étude montrent que les adolescentes pendant la grossesse, pour l'accouchement et pendant la période post-partum adoptent les mêmes itinéraires thérapeutiques quelle que soit la région visitée.

D’une manière générale, les adolescentes mariées ou non ont recours aux soins traditionnels à domicile par l'utilisation de plantes et/ou décoctions et ne se rendent au centre de santé qu'en cas de non satisfaction. Même si l'étude a montré que la plupart des adolescentes suivent régulièrement la $\mathrm{CPN}$ et accouchent à la maternité, cela ne les empêchent pas d'avoir recours aux tradipraticiens et marabouts à la recherche d'amulettes et/ou de mixtures de protection pour le nouveau-né. Les soins traditionnels donnés par les personnes ressources au post-partum et au nouveau-né viennent s’ajouter aux soins modernes reçus au centre de santé.

Une minorité d'adolescentes non mariées suivent des itinéraires différents du fait du refus de leur état ; Ce qui les amène à vouloir avorter. Cela explique le recours à l'automédication et aux tradipraticiens. Le centre de santé est le dernier recours où elles espèrent toujours faire aboutir leur projet. En cas d'échec, très peu d'entre elles recherchent le centre de santé pour le suivi de leurs grossesses. Certaines arrivent à avorter, quelques unes accouchent au CSPS et les autres à domicile. Du fait de la situation complexe dans laquelle la grossesse des adolescentes non mariées survient, il est difficile de tracer un itinéraire thérapeutique très précis de cette cible qui se cache du fait de la gêne qu'elle éprouve par rapport à sa situation.

Le suivi sanitaire des adolescentes en grossesse, à l'accouchement et pendant la période postpartum présente des avantages qui peuvent sensiblement faire réduire la mortalité maternofœtale. En effet, une grossesse d'adolescente suivie correctement et un accouchement dans un centre de santé peuvent faire éviter les complications obstétricales dont sont généralement victimes les adolescentes du fait de leur jeune âge et de leur immaturité physique. C'est ce type de suivi qui doit être encouragé au niveau des personnes ressources qui sont en charge des adolescentes au niveau familial et communautaire. Pour ce faire, des campagnes de sensibilisation doivent être dirigées vers ces personnes. De plus, les capacités en ressources humaines et matérielles de l'Action Sociale et des services de santé doivent être renforcées en vue d'accompagner les actions de ces personnes ressources et de répondre aux sollicitations des adolescentes.

De par l'intérêt et l'engouement de la population enquêtée pour le sujet, il apparaît que le contexte social est favorable à la mise en œuvre d'activités sur la SR en direction des différents groupes cibles. Les besoins à satisfaire sont réels et manifestes sur le terrain et des actions concrètes sont attendues en vue d'améliorer les conditions de vie des adolescentes, des adolescentes en grossesse et des post-partum. 



\section{RECOMMANDATIONS}

Au regard des insuffisances de connaissances par rapport à la santé sexuelle et reproductive enregistrées auprès des différentes cibles et qui limitent une prise en charge efficiente et efficace de la grossesse, de la parturiente et du bébé, découlent plusieurs recommandations adressées au projet.

\section{Sur les grossesses précoces et non désirées}

Pour réduire les grossesses précoces et non désirées, le projet doit prendre en compte les recommandations suivantes :

1- Sensibiliser les parents, les adolescentes et les personnes ressources sur la santé sexuelle et reproductive des adolescentes ;

2- Créer et/ou dynamiser les espaces réservés aux jeunes où ils peuvent se rencontrer et échanger sur la SR ;

3- Renforcer les connaissances sur la gestion de la grossesse et post-partum des personnes ressources en vue de leur permettre d'être plus efficaces.

\section{Sur la fréquentation des centres de santé}

Pour accroître le taux de fréquentation des centres de santé, l'étude recommande de:

1. Améliorer et adapter les offres et la configuration des centres de santé aux besoins des adolescentes ;

2. Rendre gratuit les frais liés à la prise en charge de la grossesse, de l'accouchement et des soins au nouveau-né ;

3. Renforcer les compétences des AV sur leurs nouveaux rôles d'accroître la fréquentation des centres de santé par les adolescentes.

\section{* Sur l'amélioration des capacités et prestations des structures de l'Action Sociale}

1- Renforcer les capacités techniques, financières et matérielles des services déconcentrés de l'Action Sociale dans la prise en charge des adolescentes (adolescentes, adolescentes en grossesse et adolescentes post-partum) ;

2- Aider les agents de l'Action Sociale à avoir un collaborateur juridique pour rendre accessible les services judiciaires et assurer le suivi au niveau de la justice des cas litigieux de grossesse précoce et non désirée.

\section{ONG /Associations}

Renforcer les capacités techniques, financières et matérielles des associations pour qu'elles assurent le rôle de mobilisation sociale. 



\section{BIBLIOGRAPHIE}

1. Adolescence et vie conjugale : Exemple de deux localités du Burkina Faso, Population Council/ UNFPA/DFID, Juin 2004.

2. Analyse situationnelle des services de santé de la reproduction au Burkina Faso, Population Council, Décembre 2006.

3. Bodo ROVOLODOMANGA, «Pour la santé et la beauté de l'enfant à naître » in grossesse et petite enfance en Afrique Noire et à Madagascar, édition Harmattan, 1991.

4. Doris BONNET, Corps biologique, corps social, procréation et maladie de l'enfant en pays mossi, Burkina Faso, édition de l’ORSTOM, 1988.

5. Etude sur les itinéraires thérapeutiques des adolescentes en grossesse et $1^{\text {ère }}$ fois parent au Bazéga et au Gourma, Population Council, Octobre 2002.

6. Georges GUIELLA, santé sexuelle et de la reproduction des jeunes au Burkina Faso : un état des lieux, 2004, série $n^{\circ} 12$.

7. Georges GUIELLA, Vanessa WOOG, Santé sexuelle et reproductive des adolescents au Burkina Faso : résultats d'une enquête nationale en 2004.

8. Ida Christiane TAMINI (thèse de doctorat de Sociologie nouveau régime "évolution et transformations des rôles et statuts des femmes en milieu rural au Burkina Faso » janvier 1999).

9. International Women's Health Coalition, sur la santé et les droits : le mariage d'enfants : un risque pour les filles de 14 ans et moins, février 2006.

10. Isabelle ANJOULAT, France LIBION, Brigitte ROSE, Les grossesses chez les adolescentes : Analyse des facteurs intervenants dans la survenue et leur issue ; Tome 2, Octobre 2007.

11. Itinéraires thérapeutiques des adolescents en grossesse ou première fois parents (Gaongo, Nafbanka, Natiaboani et Koaré), Population Council/ UNFPA/DFID, Janvier 2005.

12. Josiane CARINE, Tantchou YAKAM, Santé reproductive des jeunes : Une revue critique de la littérature. Passage, Projet d'approche solidaire en santé Génésique, janvier 2007.

13. La chronique du CEPED, la sexualité des adolescents au sahel, Avril-juin 1997, №25.

14. Laura COIN-BARIOU, Grossesse à l'adolescence : événement de vie, Avènement d'un 
sujet ? Thèse pour le diplôme d’Etat de docteur en médecine, 2005.

15. Les accoucheuses traditionnelles, déclaration conjointe OMS/FUNUAP/UNICE, 1993.

16. Mesure communication, rapport de politique générale, réduire les risques associés à la grossesse et à l'accouchement, Mai 2000.

17. Médecine et santé de l'adolescent: Acte du colloque $2^{\text {ème }}$ journée de médecine et santé de l'adolescent, 9 décembre 2000-Poitiers.

18. Projet mille jeunes filles, Adolescentes rurales : Entre espoir et réalités, Population Council, Juin 2004.

19. Projet mille jeunes filles, ces filles de la deuxième promotion, Population Council, Juin 2004.

20. Projet mille jeunes filles, Les adolescentes à l'école du développement au Burkina Faso, Population Council, Juin 2004.

21. Projet mille jeunes filles, Pour une meilleure participation au développement, Population Council, Juillet 2004.

22. Projet mille jeunes filles, Une expérience d'intégration entre promotion économique et santé de la reproduction, Population Council, Juin 2004.

23. Projet promotion de la santé sexuelle et reproductive des adolescents(e) s Rapport annuel sur le Projet BKF/05/01/11, UNFPA Bureau du Burkina Faso $5^{\text {ème }}$ programme de coopération SP-Santé de la reproduction, Janvier 2005.

24. Projet PSADO, BKF/05/01/11 Promotion de la Santé Sexuelle Reproductive des Adolescentes, Rapport de mise au 30 Octobre 2004, UNFPA Bureau du Burkina Faso SP Santé de le Reproduction, Ministère de l'action sociale et de la Solidarité Nationale, Octobre 2004.

25. Rapport de la formation des pairs éducatrices de Natiaboani et de Koaré, Population Council, fada N'gourma du 26 Avril au 7 Mai 2004.

26. Revue des politiques et des programmes sur les adolescents et les jeunes au Burkina Faso, Population Council, Janvier 2005.

27. Suzanne LALLEMAND, Grossesse et petite enfance en Afrique noire et à Madagascar, édition de l'harmattan, 1992.

28. The Allan Guttmacher Institute, Les adolescents au Burkina Faso: santé sexuelle et reproductive en bref, 2004, série $n^{\circ} 3$.

29. UNFPA, état de la population mondiale 2003. 
30. UNFPA, état de la population mondiale 2005. La promesse d'égalité : Egalité des sexes, santé en matière de procréation et objectifs du millénaire pour le développement.

31. UZAN M., PHARISIEN I., et SEINCE N., la grossesse et l'accouchement des adolescentes, hôpital Jean Verdier BONDY, JTA 2004. 